\title{
Contextualizando el derecho administrativo global
}

Fecha de recepción: 5 de abril de 2017

Fecha de aceptación: $1^{\circ}$ de noviembre de 2017

DOI: dx.doi.org/10.12804/revistas.urosario.edu.co/acdi/a.6544

\section{Sebastián López Escarcena**}

Resumen: varias son las denominaciones que se han propuesto para reemplazar a la de derecho internacional. Entre estas, se pueden mencionar los así llamados derecho cosmopolita, derecho transnacional, derecho mundial y derecho global. Desde esta perspectiva, un proyecto académico ha planteado ciertas herramientas de análisis que giran en torno a lo que se ha denominado como derecho administrativo global. Las premisas de esta aproximación parecen novedosas, pero el examen contextualizado de estas permite concluir que se acercan no solo a las de los derechos antes señalados, sino también a las de otras iniciativas académicas que han reflexionado sobre el problema del derecho más allá del Estado, como la escuela de New Haven, el constitucionalismo o los recientes proyectos sobre el ejercicio del poder público internacional y sobre la creación

* Este trabajo es parte del proyecto Fondecyt Regular N ${ }^{\circ} 1150302$, adjudicado en 2015 y titulado "Trato justo y equitativo: el buen gobierno en el derecho global".

** Profesor asociado de la Facultad de Derecho de la Pontificia Universidad Católica de Chile, e investigador asociado del Centro para el Estudio de la Gobernanza Global de la Universidad Católica de Lovaina (Bélgica) y del Instituto de Investigación en Derecho Internacional y Europeo de la Universidad de París 1 Panthéon-Sorbonne (Francia). PhD (Edimburgo), LLM (Leiden), abogado y licenciado en Derecho (Católica de Chile). Correo electrónico: rlopeze@uc.cl

Para citar este artículo: López Escarcena, S., "Contextualizando el derecho administrativo global", ACDI - Anuario Colombiano de Derecho Internacional, 2018, 11, pp. 259-305.

DOI: dx.doi.org/10.12804/revistas.urosario.edu.co/acdi/a.6544 
jurídica informal internacional, del Instituto Max Planck de Heidelberg y del Instituto de La Haya para la Internacionalización del Derecho, respectivamente. El presente artículo busca estudiar críticamente el derecho administrativo global, relacionándolo con estas otras aproximaciones que han discurrido sobre la sociedad internacional y el derecho que la regula.

Palabras clave: derecho internacional, derecho global, derecho transnacional, escuela de New Haven, solidarismo.

\section{Contextualising Global Administrative Law}

Abstract: Several names have been proposed to replace that of international law. Among these, one can mention the so-called cosmopolitan law, The transnational law, The world law and The global law. From this standpoint, an academic project has offered certain tools of analysis pivoted on what it has called global administrative law. The premises of this approach seem novel, but their contextualised examination allows us to conclude that they are close not only to the above-mentioned laws, but to other academic initiatives that have raised the issue of law beyond the state, like the New Haven School, constitutionalism or the recent projects on the exercise of international public authority of the Max Planck Institute in Heidelberg and of The Hague Institute for the Internationalization of Law, respectively. The present article aims to study global administrative law in a critical manner, relating it to these other approaches that have reflected upon the international society and the law that governs it.

Keywords: International law, global law, transnational law, New Haven school, solidarism.

Contextualizando o direito administrativo global

Resumo: várias são as denominações que se têm proposto para substituir à de direito internacional. Entre elas, podem-se mencionar os assim chamados direito cosmopolita, direito transnacional, direito mundial e direito global. Desde esta perspetiva, um projeto académico tema presentado certas ferramentas de análise que giram em torno ao que se tem denominado como direito administrativo global. As premissas desta aproximação parecem 
inovadoras, mas o exame contextualizado destas permite concluir que se aproximam não só às dos direitos antes assinalados, mas também às de outras iniciativas académicas que têm refletido sobre o problema do direito para além do Estado, como a escola de New Heaven, o constitucionalismo ou os recentes projetos sobre o exercício do poder público internacional e sobre a criação jurídica informal internacional, do Instituto Max Planck de Heidelberg e do Instituto da Haia para a Internacionalização do Direito, respetivamente, o presente artigo busca estudar criticamente o direito sobre a sociedade internacional e o direito que a regula.

Palavras-chave: direito internacional, direito global, direito transnacional, escola de New Haven, solidarismo.

\section{Introducción}

Desde mediados del siglo xx, la doctrina ha venido proponiendo distintas alternativas al derecho internacional. Se trata de iniciativas académicas destinadas a superar los problemas que presenta hoy el término que fuera acuñado por Jeremy Bentham (1748-1832) en 1780. ${ }^{1}$ Si bien los nombres y características del derecho sugerido generalmente varían de autor en autor, las diferentes teorías que se han planteado tienden a asemejarse en dos puntos principales. Por un lado, describen el fenómeno de la desaparición gradual de las tradicionales fronteras entre lo internacional y lo nacional, y entre lo público y lo privado; y, por otro, critican el sistema de fuentes y sujetos que históricamente ha regido al derecho internacional, que incluye principalmente a tratados y costumbres, así como a Estados y organizaciones internacionales. En este contexto, y a modo ilustrativo, pueden mencionarse las teorías que hablan de un derecho transnacional, de un derecho mundial, de un derecho cosmopolita y de un derecho glo$\mathrm{bal}^{2}$ La influencia que cada una de estas denominaciones ha tenido en la doctrina depende tanto del renombre de su principal exponente como

\footnotetext{
1 Ver Bentham, Jeremy, An introduction to the principles of morals and legislation, T. Payne and Son, London, 1780, cccxxvi.

2 Ver e.g. Hennebel, Ludovic, "Penser le droit administratif global. Scepticisme, idealisme et pragmatisme", en Bories, Clémentine (ed.), Un droit administratif global? A global administrative law?, Editions Pedone, París, 2012, p. 69.
} 
de la cantidad y calidad de los otros autores que han optado por propagarlas. Así, las iniciativas que se han quedado en un esfuerzo meramente individual no han logrado el impacto de aquellas que han adquirido un verdadero carácter colectivo. La más conocida de estas doctrinas tiene justamente esta particularidad. Impulsada desde la Universidad de Nueva York (NYU) por Benedict Kingsbury y Richard Stewart, tiene por objeto incentivar el estudio de un "derecho administrativo global" o GAL, por sus siglas en inglés. ${ }^{3} \mathrm{~A}$ pesar de que este proyecto ha tenido como principal promotor a la NYU, el hecho de que su manifiesto de 2005 - como lo ha llamado Lorenzo Casini- fuera escrito por un neozelandés (Kingsbury), un alemán (Nico Krisch) y un estadounidense (Stewart) demuestra que ha tenido un carácter transnacional desde sus inicios. ${ }^{4}$ En pocos años, el GAL Project se ha transformado en una auténtica escuela que reconoce dos líneas principales: una desarrollada en Norteamérica y otra en Europa occidental, de manera casi simultánea. ${ }^{5}$ Sin embargo, también han escrito sobre el GAL autores africanos, asiáticos, latinoamericanos y oceánicos. ${ }^{6}$

3 Según Stephen Neff, el principal exponente de esta corriente de pensamiento es Kingsbury.

Ver Neff, Stephen, Justice among nations: a history of international law, Harvard University Press, Cambridge, MA, 2014, p. 458.

4 Ver Casini, Lorenzo, "Global administrative law scholarship”, en Cassese, Sabino (ed.), Research handbook on global administrative law, Edward Elgar Publishing, Cheltenham, 2016, pp. 551 y $554-555$.

Ver también Kingsbury, Benedict, Krisch, Nico \& Stewart, Richard, “The emergence of global administrative law", Law and Contemporary Problems, 2005, 68, (3-4), pp. 15-61.

5 Ver Guilmain, Antoine, "Du droit cosmopolitique au droit global: pour une rupture epistemologique dans l'approche juridique", Revue Quebecoise de Droit International, 2014, 26, (2), pp. 219-236, en pp. 231-234; y Mockle, Daniel, "Le debat sur les principes et les fondements du droit administratif global", Cahiers de Droit, 2012, 53, (1), pp. 3-48, en pp. 5-10.

Ver en general Casini, "Global administrative law...”, 2016.

6 Ver e.g. (en África) Glazewski, Jan \& Rumble, Olivia, "A rose is a rose but is an 'acacia' an 'acacia'? Global administrative law in action", Acta Juridica, 2009, (1), pp. 381-402; (en Asia) Chimni, Bhupinder Singh, "Co-option and resistance: two faces of global administrative law", New York University Journal of International Law and Politics, 2005, 37, (4), pp. 799-827; (en Latinoamérica) Urueña, René, "Global administrative law and the global south", en Cassese, Sabino (ed.), Research handbook on global administrative law, Edward Elgar Publishing, Cheltenham, 2016; y (en Oceanía) McLean, Janet, "Divergent legal conceptions of the state: implications for global administrative law", Law and Contemporary Problems, 2005, 68, (3-4), pp. 167-187. 
De hecho, se ha trabajado tanto sobre el tema que incluso se han publicado bibliografías sobre derecho administrativo global, o se las ha puesto a disposición de cualquier interesado en internet. ${ }^{7}$

En la actualidad, distintas aproximaciones teóricas relacionan a la gobernanza global con la potestas o poder público socialmente reconocido. Es lo que hacen el GAL Project, el proyecto sobre el Ejercicio del Poder Público Internacional del Instituto Max Planck para el Derecho Público Comparado e Internacional y el proyecto sobre la Creación Jurídica Informal Internacional del Instituto de La Haya para la Internacionalización del Derecho, y es lo que ha venido haciendo desde hace un tiempo la doctrina del constitucionalismo. ${ }^{8}$ Todas estas visiones estudian el fenómeno del derecho más allá del Estado, y los problemas de legitimidad que esto lleva aparejado. La esencia de lo planteado por el GAL Project no solo liga al derecho administrativo global con otros derechos como el transnacional, el mundial, el cosmopolita y el simplemente global, sino que también lo conecta con otras iniciativas académicas que han reflexionado sobre la naturaleza de la sociedad internacional y el derecho que la regula. ${ }^{9}$ No es casualidad, entonces, que el GAL Project se vincule con otras corrientes doctrinales como las denominadas escuelas de New Haven y de Manhattan, cuyos nombres aluden a las localidades donde se ubican las universidades o institutos de los cuales provienen la mayoría de sus expositores. Como indica su título, este artículo pretende contextualizar el derecho administrativo global con estos otros acercamientos que han buscado entender

Ver e.g. Gunton, Dave, Livermore, Mike, Tzanakopoulos, Antonios, et al., "A global administrative law bibliography", Law and Contemporary Problems, 2005, 68, (3-4), pp. 357-377.

Ver también las bibliografías disponibles en: http://www.iilj.org/gal/resources/bibliography/ y http:/ /www.irpa.eu/en/gal-publications/, consulta del 3 de abril de 2017.

8 Ver D'Aspremont, Jean, "Droit administratif global et droit international”, en Bories, Clémentine (ed.), Un droit administratif global? A global administrative law?, Editions Pedone, París, 2012, pp. 85-87 y 92-94.

Ver también Dubin, Laurence, "Le droit administratif global, analyse critique de son existence et son articulation avec le droit international public", en Bories, Clémentine (ed.), Un droit administratif global? A global administrative law?, Editions Pedone, París, 2012, pp. 99-101.

$9 \quad$ Ver e.g. Somek, Alexander, "Administration without sovereignty", en Dobner, Petra \& Loughlin Martin (eds.), The twilight of constitutionalism?, Oxford University Press, Oxford, 2010, pp. 269-71; y Krisch, Nico, "Global governance as public authority: an introduction", International Journal of Constitutional Law, 2012, 10, (4), pp. 976-987, en pp. 982-983. 
y explicar los fenómenos antes mencionados. Con este fin, analizará brevemente no solo las teorías del derecho transnacional de Philip Jessup (1897-1986), del derecho mundial de Harold Berman (1918-2007), del cosmopolitismo de Immanuel Kant (1724-1804), del nuevo derecho global de Rafael Domingo y del nuevo ius gentium de Antonio Cançado Trindade, sino que examinará los postulados básicos del GAL Project y las principales críticas que ha recibido, y lo relacionará con el proyecto del Instituto Max Planck de Heidelberg, el constitucionalismo, el proyecto del Instituto de La Haya para la Internacionalización del Derecho y las escuelas de New Haven y de Manhattan.

\section{Más allá del derecho internacional}

Uno de los primeros en plantear una denominación alternativa al derecho internacional fue Philip Jessup, quien como profesor de la Universidad de Columbia postuló en 1956 un "derecho transnacional", destinado a regular los hechos o conductas que trascienden las fronteras estatales, incluyendo tanto al derecho internacional público como privado, así como otras reglas no incluidas en dichas categorías. Según Jessup, las situaciones transnacionales involucran a individuos, empresas, organizaciones internacionales y todo tipo de grupos de personas. ${ }^{10}$ Este derecho híbrido, mezcla de derecho interno e internacional, reconoce la importancia creciente que han adquirido los actores y las reglas no estatales en una comunidad mundial caracterizada por su complejidad. ${ }^{11}$ Años más tarde, Harold Berman propuso desde la

10 Ver en general Jessup, Philip, Transnational law, Yale University Press, New Haven, Ст, 1956.

Ver también Jessup, Philip, "Diversity and uniformity in the law of nations", American Journal of International Law, 1964, 58, (2), pp. 341-358.

Jessup no fue el primero en hablar de un derecho transnacional, pero sí fue quien desarrolló y popularizó el término.

Ver Schachter, Oscar, "Philip Jessup's life and ideas", American Journal of International Law, 1986, 80, (4), pp. 878-895, en pp. 893-894.

De hecho, Jessup reconoce que su derecho transnacional es similar, sino idéntico, al derecho intersocial unificado de Georges Scelle (1878-1961).

Ver Jessup, Transnational law, 1956, p. 3; y Jessup, "Diversity and uniformity...”, 1964, nota 4 en p. 342.

11 Ver Oliver, Covey, "Philip C. Jessup's continuing contribution to international law", Columbia Law Review, 1962, 62, (7), pp. 1132-1137, en pp. 1134-1135; y Domingo, Rafael, ¿Qué es el derecho global?, Editorial Aranzadi, Cizur Menor, Navarra, 2008, pp. 84-86. 
Universidad Emory un "derecho mundial” que comprende, pero no reemplaza, a los términos "derecho internacional” de Bentham y "derecho transnacional" de Jessup, y busca rescatar la noción de ius gentium como derecho común a la humanidad. ${ }^{12}$ Como ejemplos de derecho mundial, Berman mencionó a la lex mercatoria y la lex sportiva. ${ }^{13}$ De acuerdo con David Bederman (1961-2011), este derecho mundial sugerido por Berman no solo tendría al ius gentium como modelo histórico, sino también al ius commune y al ius canonicum. ${ }^{14}$ Como el derecho transnacional de Jessup, el derecho mundial de Berman refleja el paso de una sociedad internacional ante todo estatal a una eminentemente civil, donde el derecho no solo se hace 'desde arriba', por medio de fuentes formales Estado-céntricas, sino también 'desde abajo', a través del conjunto de interacciones que se producen en dicha sociedad. ${ }^{15}$

El nombre de "derecho mundial" utilizado por Berman no es completamente inédito. Giorgio del Vecchio (1878-1970), el conocido profesor de la Universidad de Roma, ya lo había mencionado en 1962, citando a Kotaro Tanaka (1890-1974) de la Universidad de Tokio, y relacionándolo con otro término que ha tenido mayor relieve doctrinal: el de "derecho cosmopolita". ${ }^{16}$ Cuesta definir lo que se entiende por tal, pues

Ver también Koh, Harold Hongjou, “The globalization of freedom”, Yale Journal of International Law, 2001, 26, (2), pp. 305-312; Koh, Harold Hongjou, "Why transnational law matters", Penn State International Law Review, 2006, 24, (4), pp. 745-753; y Zumbansen, Peer, "Transnational law", en Smits, Jan (ed.), Elgar encyclopedia of comparative law, Edward Elgar Publishing, Cheltenham, 2006, pp. 738-754.

12 Ver Berman, Harold, "World law", Fordham International Law Review, 1995, 18, (5), pp. 1617-1622; Berman, Harold, "The historical foundations of law", Emory Law Journal, 2005, 54, (5), pp. 13-24; y Berman, Harold, "Introduction to the World Law Institute", Emory International Law Review, 2008, 22, (1), pp. 1-6.

13 Ver Berman, "Introduction to the World Law Institute", 2008, pp. 1-2.

La lex mercatoria es también uno de los típicos ejemplos de derecho transnacional.

Ver Koh, "Why transnational law matters", 2006, pp. 746-747 y 750; Koh, "Is there a 'new' New Haven School...”, 2007, pp. 559-560; Zumbansen, “Transnational law”, 2006, pp. 740-743 y 745-746; y Sato, Yoshiaki, "Towards the institutionalization of cosmopolitan law-making”, Alberta Law Review, 2009, 46, (4), pp. 1141-1160, en pp. 1143-1144.

14 Ver Bederman, David, "World law transcendent", Emory Law Journal, 2005, 54, (5), pp. 53-77.

15 Ibid., p. 71.

16 Ver Del Vecchio, Giorgio, “Toward a world law”, Boston University Law Review, 1962, 42, (2), pp. 155-159. 
la noción de cosmopolitismo proviene principalmente de las ciencias políticas y de las relaciones internacionales, donde ha sido utilizada con los más diversos significados. De esta manera, puede haber cosmopolitismo institucional, moral o cultural. ${ }^{17} \mathrm{El}$ primero se caracteriza por promover la reforma del orden mundial y puede, a su vez, ser político, económico y legal, según abogue por una gobernanza global centralizada, por un libre mercado universal o por una justicia distributiva globalizada o un derecho único para la humanidad, estructurado sobre principios constitucionales que destacan el rol que juegan las personas no estatales. ${ }^{18}$ Cualquiera sea el adjetivo que lo acompañe, el cosmopolitismo pone al individuo como unidad central de una comunidad universal compuesta por todos los seres humanos. ${ }^{19}$ Diógenes de Sinope (412-323 a. C.) fue el primero en calificarse de cosmopolita o ciudadano del mundo en la Antigua Grecia. Más tarde, estoicos como Cicerón (106-43 a. C.) y Marco Aurelio (121-180 d. C.) hablaron en Roma de un derecho universal de la humanidad, sentando las bases del cosmopolitismo legal. Pero no fue sino hasta Immanuel Kant que el derecho cosmopolita empezó a desvincularse del ius gentium $\mathrm{y}$, por lo tanto, del ius naturale. ${ }^{20}$

17 Ver Rudall, Jason, "A cartography of cosmopolitanism: particularising the universal", Cambridge Journal of International and Comparative Law, 2014, 3, (3), pp. 747-769.

Ver también Çali, Başak, "On legal cosmopolitanism: divergences in political theory and international law", Leiden Journal of International Law, 2006, 19, (4), pp. 1149-1163, en pp. 1154-1159; y Feldman, Noah, "Cosmopolitan law?", Yale Law Journal, 2007, 116, (5), pp. 1022-1070, en pp. 1026-1030.

18 Ver Rudall, "A cartography of cosmopolitanism...”, 2014, pp. 756-764.

Ver también Çali, "On legal cosmopolitanism...”, 2006, pp. 1149-1150; y Brown, Garrett Wallace, "Moving from cosmopolitan legal theory to legal practice: models of cosmopolitan law", Legal Studies, 2008, 28, (3), pp. 430-451, en pp. 438-441.

Cf. Koskenniemi, Martti, "Legal cosmopolitanism: Tom Franck's messianic world", New York University Journal of International Law and Politics, 2003, 35, (2), pp. 471-486, en pp. 475-478.

19 Ver Çali, “On legal cosmopolitanism...”, 2006, p. 1150.

Ver también Fine, Robert, Cosmopolitanism, Routledge, Abingdon-on-Thames, 2007, pp. 2-5.

Cf. Gordon, Geoffrey, "The innate cosmopolitan tradition of international law", Cambridge Journal of International and Comparative Law, 2013, 2, (4), pp. 906-934.

20 Ver Feldman, “Cosmopolitan law?”, 2007, pp. 1026-1027 y 1030-1032; y Brown, “Moving from cosmopolitan legal theory...", 2008, pp. 432-435.

Cf. Fine, Cosmopolitanism, 2007, pp. 22-29; y Capps, Patrick, "Natural law and the law 
A fines del siglo XVIII, Kant retomó el concepto de derecho cosmopolita de los estoicos, adaptándolo al sistema westfaliano de Estados. ${ }^{21}$ De acuerdo con el célebre profesor de la Universidad de Königsberg, el derecho público presupone un Estado de derecho y puede manifestarse como ius civitatis, como ius gentium y como ius cosmopoliticum. Mientras el derecho público nacional e internacional proviene exclusivamente de órganos estatales, el derecho cosmopolita se origina en estos y en actores diferentes al Estado. ${ }^{22}$ Puesto que la humanidad está destinada a la cohabitación y la codependencia, Kant consideró necesario promover la solidaridad no solo entre los Estados, sino también entre los individuos. Esto lo hace el derecho cosmopolita a través de su principio constitucional fundamental: el de la hospitalidad universal entre los seres humanos. Al asegurar la libertad de movimiento de personas y de comercio de bienes, el ius cosmopoliticum sería el único capaz de garantizar una paz perpetua. Para lograr lo anterior, el derecho cosmopolita requiere cumplir con ciertos requisitos. Por un lado, tiene que prevalecer jerárquicamente sobre el derecho público nacional e internacional. Por otro, debe ser implementado por una foedus pacificum o federación de repúblicas que tengan una constitución democrática y separación de poderes, destinada a extenderse progresivamente a todos los Estados. ${ }^{23}$

of nations", en Orakhelashvili, Alexander (ed.), Reseach handbook on the theory and history of international law, Edward Elgar Publishing, Cheltenham, 2011, pp. 61-64 y 80-92.

21 Ver Sato, "Towards the institutionalization of...", 2009, pp. 1146-1147.

Ver en general Kant, Immanuel, Zum ewigen frieden. Ein philosophischer entwurf, Friedrich Nicolovius, Königsberg, 1795.

Hay traducción al castellano. Ver Kant, Immanuel, Hacia la pazperpetua. Un ensayo filosófico, Editorial Biblioteca Nueva, Madrid, 2016.

Si bien esta es la más conocida de las obras de Kant sobre derecho internacional, hay otros textos en los que también se refiere al derecho cosmopolita.

Ver en general Kant, Immanuel, 'Toward perpetual peace' and other writings on politics, peace, and history, Yale Unversity Press, New Haven, 2006.

Ver también Tesón, Fernando, "The Kantian theory of international law", Columbia Law Review, 1992, 92, (1), pp. 53-102.

22 Ver Brown, "Moving from cosmopolitan legal theory...", 2008, p. 435; Sato, “Towards the institutionalization of...", 2009, p. 1145; Guilmain, "Du droit cosmopolitique au droit global...", 2014, pp. 227-228; y Rudall, “A cartography of cosmopolitanism...”, 2014, pp. 762-763.

23 Ver Brown, "Moving from cosmopolitan legal theory...", 2008, pp. 434-438 y 445-450; Sato, "Towards the institutionalization of...", 2009, pp. 1145-1147; Domingo, ¿Qué es el derecho global?, 2008, pp. 70-72; Guilmain, "Du droit cosmopolitique au droit global...", 
Sin perjuicio de la indiscutible influencia que Kant ha tenido en el desarrollo del derecho internacional, la denominación alternativa que ha logrado mayor aceptación en la doctrina es la de 'derecho global', que a su vez sostiene múltiples aproximaciones teóricas. ${ }^{24}$ Está, por ejemplo, la de Rafael Domingo, quien habla de un "nuevo derecho global" que encuentra su fundamento en el individuo y que se centra en la dignidad humana. Con no poco voluntarismo, este profesor de la Universidad de Navarra ha propuesto un derecho cuyos principios informadores son aquellos comunes a cualquier ordenamiento jurídico, como la justicia, la razonabilidad y la coerción, y aquellos específicos de este nuevo ordenamiento global, que lo distinguen del internacional, como la universalidad, la solidaridad, la subsidiariedad y la horizontalidad o democratización. ${ }^{25}$ De formación romanista, quizá lo más interesante del libro de Domingo donde expuso su teoría en extenso, es su relato histórico sobre la evolución del ius gentium desde la Antigua Roma hasta nuestros días, pasando por las distintas fases que los nombres 'derecho de gentes' y 'derecho internacional' cubren a

2014, pp. 220-221 y 226-228; y Rudall, “A cartography of cosmopolitanism...”, 2014, pp. $762-764$.

24 Pueden encontrarse aspectos del cosmopolitismo de Kant en la obra de Hersch Lauterpacht (1897-1960), Hans Kelsen (1881-1973), Philip Allott y Jürgen Habermas, entre otros.

Ver Çali, “On legal cosmopolitanism...”, 2006, pp. 1155-1156; Guilmain, "Du droit cosmopolitique au droit global...", 2014, pp. 226-230; y Rudall, “A cartography of cosmopolitanism...", 2014, p. 764.

Ver también Tesón, “The Kantian theory...”, 1992, nota 7 en p. 54 y pp. 55-56; Koskenniemi, "Legal cosmopolitanism...", 2003, pp. 471-475; y Fine, Cosmopolitanism, 2007, pp. 2-3, 22 y 59-77.

Por ejemplo, el proyecto kantiano de una federación de Estados democráticos que promuevan el libre tránsito de personas e intercambio de bienes se asemeja mucho a la iniciativa que hoy se conoce como Unión Europea.

Ver Brown, "Moving from cosmopolitan legal theory...", 2008, pp. 436-438.

25 Ver en general Domingo, ¿Qué es el derecho global?, 2008.

Este libro fue traducido al inglés, con algunas adiciones. Ver Domingo, Rafael, The new global law, Cambridge University Press, Nueva York, 2010.

Cf. Hennebel, "Penser le droit administratif global...", 2012, pp. 73-76, 78 y 80; y Al Hajjaji, Shams Al Din, "Review of The New Global Law by Rafael Domingo", Berkeley Journal of International Law, 2014, 32, (1), pp. 268-280.

Ver también Domingo, Rafael, "The crisis of international law", Vanderbilt Journal of Transnational Law, 2009, 42, (5), pp. 1543-1593; Domingo, Rafael, "Gaius, Vattel, and the new global law paradigm”, European Journal of International Law, 2011, 22, (3), pp. 627-647; y Domingo, Rafael, “The new global human community", Chicago Journal of International Law, 2012, 12, (2), pp. 563-587. 
grandes rasgos. ${ }^{26}$ En su afán de devolverle el papel protagónico a las personas y sus derechos subjetivos, que perdieran en 1648 tras la instauración del esquema que privilegia a los Estados y su soberanía, así como en su cariz naturalista, el proyecto de Domingo recuerda en algo el "nuevo ius gentium" planteado por Antonio Cançado Trindade, profesor emérito de la Universidad de Brasilia. Este nuevo derecho de gentes vendría a recoger la esencia de los postulados de autores fundacionales del derecho internacional, como Francisco de Vitoria (1483-1546), Francisco Suárez (1548-1617), Hugo Grocio (1583-1645), Alberico Gentili (1552-1608) y Christian Wolff (1679-1754), para así reemplazar al ius inter gentes que adquiriera relevancia a partir de cierta doctrina y jurisprudencia internacional de los siglos XIX y xx. ${ }^{27}$ Con una salvedad: Cançado Trindade no solo propone un nuevo ius gentium que incluya únicamente a los individuos, sino también a los pueblos y a la humanidad como un todo, que en su opinión conforman la comunidad internacional o civitas maxima gentium. Desde esta perspectiva, el planteamiento de Cançado Trindade se acercaría al cosmopolitismo, en una particular variante que Geoffrey Gordon ha apellidado como innato. ${ }^{28}$

\section{Global administrative law, diritto amministrativo globale, etc.}

El término 'derecho administrativo global' fue utilizado por primera vez en 2002, en un simposio de la Administrative Law Review. ${ }^{29}$ No obstante, fueron Benedict Kingsbury y Richard Stewart, junto a Nico Krisch, hoy del Instituto de Altos Estudios Internacionales de Ginebra, quienes definieron por primera vez el derecho administrativo global en Nueva York, algunos años después. ${ }^{30}$ Usualmente, se considera que la publicación de

\footnotetext{
26 Ver Domingo, ¿Qué es el derecho global?, 2008, pp. 33-102.

27 Ver en general Trindade, Antonio Cançado, International law for humankind: towards a new jus gentium, Martinus Nijhoff Publishers, Leiden, 2010.

Este libro fue traducido al francés. Ver Trindade, Antonio Cançado, Le droit international pour la personne bumaine, Editions Pedone, París, 2011.

28 Ver Gordon, “The innate cosmopolitan tradition...”, 2013.

29 Ver Casini, “Global administrative law...”, 2016, p. 549.

Ver también Koch, Charles, "Introduction: globalization of administrative and regulatory practice”, Administrative Law Review, 2002, 54, (1), pp. 409-414, en p. 411.

30 Ver Kingsbury, Krisch \& Stewart, "The emergence of global administrative law", 2005, p. 17.
} 
Kingsbury, Krisch y Stewart de 2005 dio inicio al estudio de este derecho. Se tiende a olvidar, sin embargo, que para entonces Sabino Cassese ya había comenzado en la Universidad de Roma su estudio del derecho más allá del Estado, que posteriormente llamaría derecho administrativo global. ${ }^{31}$ Aun cuando Cassese no fue el único autor europeo en proponer, con anterioridad al mencionado artículo de Kingsbury, Krisch y Stewart, que los desafíos planteados por la globalización sean analizados desde el derecho administrativo, sí ha sido el más prolífico. ${ }^{32}$ Provengan de Norteamérica o de Europa occidental, los miembros del GAL Project coinciden generalmente en que todo ejercicio de autoridad que tenga un impacto en actores tanto públicos como privados constituye una materia de estudio pertinente. ${ }^{33}$ El proyecto postula, en lo esencial, que hay un derecho compuesto por ciertos principios y normas que son aplicables a procesos de naturaleza administrativa que involucran estructuras jurídico-políticas que exceden a las puramente intraestatales. ${ }^{34}$ De esta manera, el manifiesto de 2005 definió al derecho administrativo global como "aquél que incluye los mecanismos, principios, prácticas y los acuerdos sociales que los respaldan y que promueven o que de otra forma afectan la accountability de los órganos globales administrativos, en particular asegurándose de que cumplan con los estándares adecuados de transparencia, participación, toma de

Este artículo fue traducido al francés y al castellano. Ver Kingsbury, Benedict, Krisch, Nico \& Stewart, Richard, "L'emergence d'un droit administratif global”, en Bories, Clémentine (ed.), Un droit administratif global? A global administrative law?, Editions Pedone, París, 2012; y Kingsbury, Benedict, Krisch, Nico \& Stewart, Richard, "El surgimiento del derecho administrativo global", Revista de Derecho Público (de la Universidad de los Andes, Colombia), 2010, (24), pp. 4-46.

31 Ver Cassese, Sabino, "Administrative law without the state - The challenge of global regulation", New York University Journal of International Law and Politics, 2005, 37, (4), pp. 663-694.

Ver también Cassese, Sabino, "Global standards for national administrative procedure", Law and Contemporary Problems, 2005, 68, (3-4), pp. 109-126.

32 Ver e. g. Auby, Jean-Bernard, La globalisation, le droit et l'état, Éditions Montchrestien, París, 2003.

Ver también Casini, "Global administrative law...", 2016, pp. 551-553 y 568.

33 Ver D'Aspremont, "Droit administratif global et droit international”, 2012, pp. 88-90.

34 Ver Kingsbury, Benedict \& Donaldson, Megan, "Global administrative law", en Wolfrum, Rüdiger (ed.), Max Planck encyclopedia of public international law, Oxford University Press, Oxford, 2015, párrafo 1. 
decisiones razonada, y legalidad, y promoviendo la efectiva evaluación de las normas y decisiones que aprueban". 35

Como puede apreciarse, el derecho administrativo global nació como un método para analizar regulaciones parcialmente ignoradas por el derecho internacional. ${ }^{36}$ Para esto, el GAL Project ha propuesto partir no del derecho internacional, sino del derecho administrativo, a fin de utilizar algunas de las categorías de este derecho para entender y explicar los problemas que surgen de la globalización. ${ }^{37}$ Esta circunstancia explica, en parte, el desarrollo de la escuela del GAL en Norteamérica y Europa occidental. Mientras que en Estados Unidos es más pragmática, privilegiando los aspectos procesales del derecho administrativo global, en Italia y Francia, así como en España, es más teórica, estudiando preferentemente lo sustantivo de este derecho. ${ }^{38}$

La noción de GAL incluye a la de derecho administrativo internacional, pero no se agota en este. ${ }^{39}$ Benedict Kingsbury ha reconocido abierta-

35 Kingsbury, Krisch \& Stewart, "El surgimiento del derecho administrativo global", 2010, p. 6.

Ver Kingsbury, Krisch \& Stewart, "The emergence of global administrative law", 2005, p. 17.

Ver también Kingsbury, Benedict, Krisch, Nico, Stewart, Richard \& Wiener, Jonathan, "Global governance as administration - National and transnational approaches to global administrative law", Law and Contemporary Problems, 2005, 68, (3-4), pp. 1-13, en p. 5; Kingsbury, Benedict, "The administrative law frontier in global governance", American Society of International Law Proceedings, 2005, (99), pp. 143-153, en p. 143; y Kingsbury, Benedict, "Weighing global regulatory rules and decisions in national courts", Acta Juridica, 2009, (1), pp. 90-119, en pp. 91-92.

36 Ver Kingsbury, Krisch \& Stewart, "The emergence of global administrative law", 2005, pp. 16-18.

Ver también Kingsbury \& Donaldson, “Global administrative law", 2015, párrafos 10-19.

37 Ver Casini, Lorenzo, "Beyond drip-painting? Ten years of GAL and the emergence of a global administration", International Journal of Constitutional Law, 2015, 13, (20), pp. 473477, en pp. 473-474 y 477; y Casini, “Global administrative law...”, 2016, pp. 548-553 y 555-556.

38 Ver Casini, “Global administrative law...”, 2016, pp. 555-560.

39 Ver ibid., pp. 549-553.

Ver también Mockle, "Le debat sur les principes et les fondements...”, 2012, pp. 5-10; y Guilmain, "Du droit cosmopolitique au droit global...", 2014, pp. 231-234.

Respecto al derecho administrativo internacional, el derecho internacional administrativo, el derecho de la función pública internacional, el derecho de las organizaciones 
mente la influencia que han ejercido en la teoría del derecho administrativo global autores como Lorenz von Stein (1815-1890), de la Universidad de Viena, y Paul Reinsch (1869-1923) y Pitman Potter (1892-1981), de la Universidad de Wisconsin-Madison, entre otros. ${ }^{40}$ Por esta razón, una de las primeras líneas de estudio del GAL Project fue el análisis de una o más organizaciones internacionales, o de estas en general, desde la nueva perspectiva propuesta. ${ }^{41}$ Otra de estas líneas fue el análisis de la práctica procesal de los órganos globales desde la tradición administrativa estadounidense, centrándose en ciertos principios adjetivos del GAL, como la participación, la transparencia, la motivación jurídica, la revisión judicial y la rendición de cuentas y responsabilidad, en el sentido de la expresión inglesa "accountability". 42 En esta primera etapa del proyecto, principios sustantivos del GAL, como la proporcionalidad y el respeto de las expectativas legítimas, fueron mencionados excepcionalmente, y muy al pasar. ${ }^{43}$

internacionales o derecho institucional internacional, y el derecho administrativo global, ver en general Bories, Clémentine, "Histoire des phénomènes administratifs au-delà de la sphère étatique: tâtonnements et hésitations du droit et/ou de la doctrine", en Bories, Clémentine (ed.), Un droit administratif global? A global administrative law?, Editions Pedone, París, 2012. 40 Ver Kingsbury, “The administrative law frontier...”, 2005, p. 143; Kingsbury, Krisch \& Stewart, "The emergence of global administrative law", 2005, pp. 19 y 27-28; Kingsbury, Benedict, "The concept of 'law' in global administrative law", European Journal of International Law, 2009, 20, (1), pp. 23-57, en pp. 23-24; y Kingsbury, Benedict \& Casini, Lorenzo, "Global administrative law dimensions of international organizations law", International Organizations Law Review, 2009, 6, (2), pp. 319-358, en p. 327.

Ver también Neff, Justice among nations..., 2014, pp. 289-290, 377 y 458.

Además de Von Stein, Reinsch y Potter, Benedict Kingsbury ha mencionado a otros profesores, de distintas universidades, que escribieron a fines del siglo XIX y principios del xx sobre el derecho administrativo internacional, como Pierre Kazansky (Odesa), Paul Négulesco (Bucarest), Karl Strupp (Fráncfort) y Karl Neumeyer (Múnich).

Ver Kingsbury, Krisch \& Stewart, "The emergence of global administrative law", 2005, pp. 19-20, 24, 26, 28-29 y 40; Kingsbury, “The administrative law frontier...”, 2005, pp. 143-144; Kingsbury \& Casini, "Global administrative law dimensions of...", 2009, p. 327; y Kingsbury \& Donaldson, "Global administrative law”, 2015, párrafos 1 y 4-8.

${ }^{41}$ Ver e. g. Cassese, "Administrative law without the state...", 2005; Cassese, "Global standards for...”, 2005; y Kingsbury \& Casini, "Global administrative law dimensions of...", 2009 .

42 Ver e. g. Stewart, Richard, "U.S. administrative law: a model for global administrative law?", Law and Contemporary Problems, 2005, 68, (3-4), pp. 63-108; y Stewart, Richard, "The global regulatory challenge to U.S. administrative law", New York University Journal of International Law and Politics, 2005, 37, (4), pp. 695-762.

43 Ver e. g. Kingsbury, Krisch \& Stewart, "The emergence of global administrative law", 
Según Kingsbury, se escogió el término 'global' para denominar este derecho a fin de destacar la inclusión de sujetos y fuentes que no están entre las generalmente aceptadas en el derecho internacional. ${ }^{44}$ Anticipándose a Antonio Cançado Trindade, este autor neozelandés agregó que el derecho administrativo global tendría que ser visto como parte de un nuevo ius gentium, más que como perteneciente al modelo tradicional de ius inter gentes. ${ }^{45}$ Es por esto que el GAL reconoce como fuentes no solo al derecho internacional público, sino también a: una versión renovada del ius gentium, descrito como una especie de lex mercatoria; un así denominado derecho público internacional, que regula el ejercicio de autoridad de este tipo; el derecho público o administrativo nacional, en perspectiva comparada; y las normas generadas por sistemas autónomos, tal como los entiende el famoso profesor de la Universidad de Bielefeld, Niklas Luhmann (1927-1998). ${ }^{46}$ En cuanto a los sujetos, para el GAL Project la índole administrativa de una actividad puede proceder de su naturaleza, efectos $\mathrm{u}$ objetivo, e incluso del tipo de entidad que la realiza. ${ }^{47}$ Así, la administración

2005, pp. 40-41; Kingsbury, “The administrative law frontier...”, 2005, pp. 144, 146-147 y 149; Cassese, "Administrative law without the state...", 2005, pp. 684-694; y Cassese, "Global standards for...", 2005, pp. 110-111, 120-121 y 125-126.

44 Ver Kingsbury, “The administrative law frontier...”, 2005, p. 144.

Ver también Kingsbury, Krisch, Stewart \& Wiener, "Global governance as administration...", 2005, p. 5; Cassese, “Administrative law without the state...”, 2005, nota 43 en pp. 669-670; Krisch, Nico \& Kingsbury, Benedict, "Global governance and global administrative law in the international legal order", European Journal of International Law, 2006, 17, (1), pp. 1-13, en p. 5; Kingsbury, “The concept of 'law'...", 2009, pp. 25-26; Kingsbury, "Weighing global regulatory rules and decisions...", 2009, p. 92; Cassese, Sabino, "Global administrative law: the state of the art", International Journal of Constitutional Law, 2015, 13, (2), pp. 465468, en p. 466; y Kingsbury \& Donaldson, “Global administrative law”, 2015, párrafo 1.

45 Ver Kingsbury, “The administrative law frontier...”, 2005, pp. 143 y 147-149.

Ver también Kingsbury, Krisch \& Stewart, "The emergence of global administrative law", 2005, pp. 29-30; y Krisch \& Kingsbury, "Global governance and global administrative law...", 2006, pp. 12-13.

46 Ver Kingsbury, Krisch \& Stewart, "The emergence of global administrative law”, 2005, pp. 29-31; Kingsbury, “The administrative law frontier...”, 2005, pp. 146-149; Kingsbury, "Weighing global regulatory rules and decisions...", 2009, pp. 92-93; y Kingsbury \& Donaldson, "Global administrative law", 2015, párrafos 20-25.

Ver también Kingsbury, “The concept of 'law'...”, 2009, pp. 52-55.

Ver en general Luhmann, Niklas, Soziale systeme: Grundriß einer allgemeinen theorie, Suhrkamp Verlag, Frankfurt, 1984.

47 Ver Kingsbury \& Donaldson, “Global administrative law”, 2015, párrafos 11-9. 
global puede provenir de organizaciones intergubernamentales formales, que son las instituciones internacionales por excelencia, como la ONU o la OEA; de organizaciones híbridas público-privadas o entidades privadas que ejercen funciones públicas, como la Organización Internacional de Normalización (ISO, por sus siglas en inglés); de redes transgubernamentales y transnacionales, que son formas de gobernanza menos estructuradas entre Estados, organizaciones internacionales y/u otros actores, como el Comité de Supervisión Bancaria de Basilea; y de formas más complejas de gobernanza, como los regímenes regulatorios globales híbridos, multinivel o informales, como los procesos de toma de decisión en materia de pesca que establece la Convención de Patrimonio Mundial. ${ }^{48}$

$\mathrm{Al}$ respecto, Kingsbury ha insistido en la noción de "publicness", en lugar de: i. e., el carácter de público que debe tener un determinado régimen regulatorio para que pueda ser considerado como parte del derecho administrativo global. Esto, en el entendido de que público es lo que proviene de la totalidad de la sociedad respectiva y se refiere a asuntos de su interés general. Los principios que componen esta condición de publicness son, entre otros: legalidad, racionalidad, proporcionalidad, preeminencia del derecho o rule of law $\mathrm{y}$ respeto de los derechos humanos. ${ }^{49} \mathrm{El}$ fundamento teórico de la noción de publicness, en virtud de la cual Kingsbury ha calificado al GAL como derecho interpúblico, estaría en una versión actualizada del concepto de derecho propuesto por H. L. A. Hart (1907-1992) de la Universidad de Oxford, complementada con ciertos criterios enunciados por Lon Fuller (1902-1978) de la Universidad Harvard. ${ }^{50}$

48 Ver Casini, "Beyond drip-painting?...”, 2015, pp. 475-477.

Ver también Kingsbury, Krisch \& Stewart, "The emergence of global administrative law", 2005, pp. 16-27 y 31-37; Kingsbury, Krisch, Stewart \& Wiener, "Global governance as administration...", 2005, p. 5; y Kingsbury, “The administrative law frontier...”, 2005, pp. 143-148; Stewart, "U.S. administrative law...”, 2005, pp. 63-68; Stewart, "The global regulatory challenge...", 2005, pp. 699-703; y Krisch \& Kingsbury, "Global governance and global administrative law...", 2006, pp. 2-5.

49 De acuerdo con Kingsbury, el principio de legalidad consiste en que los actores de un sistema deban actuar conforme a las reglas que lo regulan; el principio de racionalidad se relaciona con el de motivación jurídica; la preeminencia del derecho tiene una naturaleza procesal; y el respeto a los derechos humanos se refiere al contenido sustantivo que no queda cubierto por la noción de rule of law.

Ver Kingsbury, “The concept of 'law'...”, 2009, pp. 31-33; y Kingsbury, "Weighing global regulatory rules and decisions...", 2009, pp. 113-118.

50 Ver Kingsbury, “The concept of 'law'...”, 2009, pp. 27-31 y 34-57. 


\section{A favor y en contra del derecho administrativo global}

La perspectiva propuesta por el GAL permitiría analizar, y eventualmente superar, la falta de legitimidad democrática y accountability de muchos de los órganos que pueblan lo que esta escuela llama el espacio administrativo global, donde las clásicas distinciones entre lo nacional e internacional, y lo público y privado, no son de utilidad..$^{51}$ Para este fin, el GAL Project ha propuesto ciertos principios, como las señaladas participación procesal, transparencia, motivación jurídica, revisión judicial y accountability en general, por un lado; y proporcionalidad y respeto de las expectativas legítimas, por otro. ${ }^{52}$ De esta manera, el espacio administrativo global pasaría a constituir un orden binario, en el que diferentes sistemas jurídicos coexisten con un grupo de principios comunes, tal como lo hicieran los derechos locales europeos con el derecho romano vulgarizado o derecho común en la Edad Media. ${ }^{53}$ Es la existencia de una global polity emergente, en la

Ver en general Hart, Herbert Lionel Adolphus, The concept of law, Oxford University Press, Oxford, 1961; y Fuller, Lon, The morality of law, Yale University Press, New Haven, Ст, 1964.

51 Ver Kingsbury, Krisch \& Stewart, "The emergence of global administrative law”, 2005, pp. 23-27; Kingsbury, Krisch, Stewart \& Wiener, "Global governance as administration...", 2005, pp. 2-5; Stewart, "U.S. administrative law...", 2005, pp. 68-71; Stewart, "The global regulatory challenge...", 2005, pp. 703-709; Krisch \& Kingsbury, "Global governance and global administrative law...", 2006, pp. 1-5 y 10-3; Kingsbury, "The concept of 'law'...", 2009, pp. 24-26; y Kingsbury, "Weighing global regulatory rules and decisions...", 2009, p. 91.

52 Ver e. g. Kingsbury, Krisch \& Stewart, "The emergence of global administrative law", 2005, pp. 16-17, 26-29 y 37-42; Kingsbury, "The administrative law frontier...", 2005, pp. 146-147; Cassese, "Administrative law without the state...", 2005, pp. 684-694; Cassese, "Global standards for...", 2005, pp. 110-111, 120-121 y 125-126; Kingsbury, "The concept of 'law'...", 2009, pp. 41-50; Kingsbury, "Weighing global regulatory rules and decisions...", 2009, p. 92; Kingsbury, “The concept of 'law'...”, 2009, pp. 23-26; y Kingsbury \& Donaldson, "Global administrative law”, 2015, párrafos 10 y 26-54.

Sobre el GAL y la accountability, ver Krisch, Nico, "The pluralism of global administrative law", European Journal of International Law, 2006, 17, (1), pp. 247-278; y Dyzenhaus, David, "Accountability and the concept of (global) administrative law", Acta Juridica, 2009, (1), pp. 3-31.

Ver también Mockle, "Le debat sur les principes et les fondements...", 2012, pp. 23-27.

53 Ver Cassese, Sabino, "The globalization of law", New York University Journal of International Law and Politics, 2006, 37, (4), pp. 973-993, en pp. 986-990 y 992-993.

Ver también Cassese, "Global administrative law...", 2015, p. 468. 
cual se ha desarrollado y opera hoy el GAL, lo que permitiría que conviva esta iura particularia con este ius commune. ${ }^{54}$ Quizás el aporte primordial de la escuela del GAL ha sido justamente la aplicación de tales principios, en particular aquellos de naturaleza administrativo-procesal, como la participación, transparencia y motivación jurídica, a la toma de decisiones en las organizaciones internacionales. ${ }^{55}$ En este sentido, el GAL no es solo un proyecto descriptivo, sino también prescriptivo. ${ }^{56}$ Vale decir, es un proyecto normativo que entiende la gobernanza global o transnacional como administración o regulación. ${ }^{57}$ Como Nico Krisch ha reconocido, el enfoque normativo del GAL es restringido. Por esta razón, se asemeja a los primeros pasos dados por los sistemas de derecho administrativo europeo en el siglo XIX, la mayoría de los cuales se tuvieron que desarrollar en ambientes difíciles, por lo general autoritarios, lo que limitó sus avances a ciertas áreas o asuntos puntuales. ${ }^{58}$

54 Ver Chiti, Edoardo, "Where does GAL find its legal grounding?", International Journal of Constitutional Law, 2015, 13, (2), pp. 486-491, en pp. 489-490.

Ver en general Cassese, Sabino, The global polity: global dimensions of democracy and the rule of law, Global Law Press, Sevilla, 2012.

Lorenzo Casini tradujo el término global polity como régimen político global en el prefacio de la traducción italiana de este libro de Cassese.

Ver Cassese, Sabino, Chi governa il mondo?, Il Mulino, Bologna, 2013.

55 Ver Casini, “Beyond drip-painting?...”, 2015, p. 474.

56 Ver Dubin, "Le droit administratif global...”, 2012, pp. 93-98.

Ver también Urueña, “Global administrative law...”, 2016, p. 392.

57 Ver Kingsbury, Krisch \& Stewart, "The emergence of global administrative law”, 2005, pp. 15-20, 25-31 y 42-59; Kingsbury, Krisch, Stewart \& Wiener, "Global governance as administration...”, 2005, pp. 2-5; Stewart, “U.S. administrative law...”, 2005, pp. 63-64 y 71-74; Kingsbury, "The administrative law frontier...", 2005, pp. 143 y 147-153; Krisch \& Kingsbury, "Global governance and global administrative law...", 2006, pp. 1-5; Kingsbury, "The concept of 'law'...", 2009, pp. 24-26; y Kingsbury, "Weighing global regulatory rules and decisions...", 2009, p. 92.

Ver también Stewart, Richard, "The normative dimensions and performance of global administrative law", International Journal of Constitutional Law, 2015, 13, (2), pp. 499-506, en pp. 500-505.

Ver en general Benvenisti, Eyal, The law of global governance, Brill-Nijhoff, Leiden, 2014.

58 Ver Krisch, Nico, "Global administrative law and the constitutional ambition", en Dobner, Petra \& Loughlin, Martin (eds), The twilight of constitutionalism?, Oxford University Press, Oxford, 2010, pp. 257-258.

Ver también Mockle, "Le debat sur les principes et les fondements...", 2012, pp. 15-16. 
La línea de estudio propuesta por el GAL Project ha sido particularmente exitosa. Entre los factores que explican esto, se puede mencionar el empleo de la palabra de mode 'global' y, sobre todo, su aproximación pragmática y casuística. En efecto, los trabajos provenientes de esta vertiente generalmente no se quedan en abstracciones doctrinales. Un buen ejemplo de esto se puede encontrar en el voluminoso libro de casos o casebook del GAL, editado por el Instituto de Investigación en Administración Pública, fundado en 2004 por Sabino Cassese y otros profesores italianos de derecho administrativo. ${ }^{59} \mathrm{~A}$ través de un método multidisciplinario, las publicaciones que se identifican con el GAL Project han analizado, desde su particular punto de vista, el accionar de distintos regímenes regulatorios, lo que a su vez ha facilitado el estudio del derecho administrativo global en diversas universidades, principalmente norteamericanas, europeas y latinoamericanas, favoreciendo la creación de redes académicas. ${ }^{60} \mathrm{~A}$ pesar de lo anterior, el GAL Project ha sido criticado y, en algunos casos, no sin buenas razones. Uno de los primeros en cuestionar el GAL fue Bhupinder Singh Chimni, quien lo hizo desde la perspectiva de la escuela de las Aproximaciones del Tercer Mundo al Derecho Internacional. ${ }^{61}$ Según este autor indio, el GAL proviene de una clase capitalista transnacional que busca legitimizar, en su propio beneficio, un derecho de naturaleza imperial. En su opinión, el GAL solo pasaría a constituir un instrumento de resistencia y cambio en el derecho internacional si sus principios procesales se complementan con

59 Ver en general Cassese, Sabino, Carotti, Bruno, Casini, Lorenzo, et al. (eds.), Global administrative law: the casebook, Istituto di Ricerche sulla Pubblica Amministrazione-Institute for International Law and Justice, Roma-Nueva York, 2012.

60 Ver Casini, “Global administrative law...”, 2016, pp. 554-563.

Ver también Möllers, Christoph, "Ten years of global administrative law", International Journal of Constitutional Law, 2015, 13, (2), pp. 469-472, en pp. 469-470.

Sobre la metodología del GAL, ver D’Aspremont, "Droit administratif global et droit international”, 2012, pp. 88-92.

Ver también Dubin, "Le droit administratif global...", 2012, pp. 96-98.

${ }^{61}$ Más conocida por la sigla TWAIL, proveniente de su nombre en inglés: Third World approaches to international law.

Sobre Los TWAIL, ver en general Mutua, Makau, "What is TWAIL?", American Society of International Law Proceedings, 2000, (94), pp. 31-40; Chimni, Bhupinder Singh, "Third World approaches to international law: a manifesto", International Community Law Review, 2006, 8, (1), pp. 3-27; y Gathii, James Thuo, "TWAIL: a brief history of its origins, its decentralized network and a tentative bibliography", Trade, Law and Development, 2011, 3, (1), pp. 26-64. 
un régimen sustantivo cercano a los derechos humanos, implementado por movimientos sociales globales y organizaciones no gubernamentales: los únicos actores capaces de hacerlo, allí donde el poder está desigualmente distribuido. ${ }^{62}$ Susan Marks y Carol Harlow han compartido, en gran medida y con otras expresiones, las críticas formuladas por Chimni. ${ }^{63}$ De acuerdo con Harlow, el GAL no debiera desentenderse de los principios fundantes del derecho administrativo comparado, en un contexto de pluralismo jurídico. Esto es, de la democracia y la preeminencia del derecho o rule of law, en su acepción amplia —i. e., tanto procesal como sustantiva- ${ }^{64}$ Kingsbury, Krisch y Stewart, los autores del manifiesto GAL de 2005, han reconocido estos cuestionamientos en más de una oportunidad. ${ }^{65}$

Un poco después de que se plantearan las primeras críticas al GAL Project, se produjo un breve debate doctrinal a partir de la noción de publicness propuesta por Kingsbury. ${ }^{66}$ Como señala Casini, esta discusión sobre el carácter jurídico del GAL se entiende mejor en el contexto del common law, ya que en los sistemas jurídicos pertenecientes al derecho continental las nociones de recht, diritto, droit y derecho tienen un significado más amplio que law. ${ }^{67}$ Por este motivo, solo excepcionalmente autores

${ }^{62}$ Ver en general Chimni, “Co-option and resistance...", 2005.

63 Ver Marks, Susan, "Naming global administrative law", New York University Journal of International Law and Politics, 2005, 37, (4), pp. 995-1001; y Harlow, Carol, "Global administrative law: the quest for principles and values", European Journal of International Law, 2006, 17, (1), pp. 187-213.

64 Ver en general Harlow, "Global administrative law...", 2006.

Ver también Harlow, Carol, "Voices of difference in a plural community", American Journal of Comparative Law, 2002, 50, (2), pp. 339-367.

65 Ver Kingsbury, Krisch \& Stewart, "The emergence of global administrative law”, 2005, pp. 27 y 51-52; Kingsbury, “The administrative law frontier...", 2005, p. 150; Stewart, "The global regulatory challenge...", 2005, p. 757; y Krisch \& Kingsbury, "Global governance and global administrative law...", 2006, pp. 11-12.

Ver también Kingsbury \& Donaldson, “Global administrative law”, 2015, párrafo 58; y Stewart, "The normative dimensions and performance...", 2015, p. 499.

${ }^{66}$ Ver e.g. Kuo, Ming-Sung, "The concept of 'law' in global administrative law: a reply to Benedict Kingsbury", European Journal of International Law, 2010, 20, (4), pp. 997-1004; y Somek, Alexander, "The concept of 'law' in global administrative law: a reply to Benedict Kingsbury”, European Journal of International Law, 2010, 20, (4), pp. 985-995.

Cf. Kingsbury, "The concept of 'law'...", 2009.

67 Ver Casini, “Global administrative law...., 2016, p. 564. 
de formación romano-germánica han terciado en esta controversia. ${ }^{68}$ De cualquier manera, no es común que los autores del GAL se ocupen de su carácter jurídico. Esto los enfrentaría al difícil asunto de conceptualizar el derecho, desviándolos de sus objetivos pragmáticos — por ejemplo, el de aumentar la participación procesal, la transparencia, la motivación jurídica, la revisión judicial y la accountability- ${ }^{69}$ Dentro de los cuestionamientos que ha recibido el GAL Project, no pueden dejar de nombrarse su falta de novedad dogmática y su poca claridad respecto a los límites del análisis propuesto. En cuanto a la primera de dichas críticas, Mathias Forteau ha señalado que los principios administrativos propuestos por el GAL no tienen nada de nuevos, ya que en gran medida fueron planteados a inicios del siglo xx por la doctrina administrativa. ${ }^{70}$ Laurence Dubin ha agregado que el derecho internacional sí ofrece herramientas de análisis para ciertas regulaciones que, según los cultores del derecho administrativo global, solo vendrían a ser inteligibles a través del método propuesto por el GAL Project. ${ }^{71}$ En relación con la segunda de tales objeciones, Joseph Weiler ha destacado el hecho de que muchas de las publicaciones académicas que usan el nombre de 'GAL' lo hacen sin que su contenido tenga mayormente que ver con el derecho administrativo global, diluyendo así los postulados de la escuela. ${ }^{72}$ Sobre este punto, Casini ha subrayado la circunstancia de que varios autores jóvenes han recurrido al GAL por dos razones equivocadas: para explicar fenómenos viejos que no necesitan de este para ser comprendidos, o porque creen que al utilizar dicho término podrán resol-

68 Ver e.g. D’Aspremont, "Droit administratif global et droit international", 2012, pp. 91-92; Dubin, "Le droit administratif global...", 2012, pp. 106-113; y Mockle, "Le debat sur les principes et les fondements...", 2012, pp. 34-44.

69 Ver Dyzenhaus, “Accountability and the concept of...”, 2009, pp. 6, 8 y 10.

Ver también Somek, "Administration without sovereignty", 2010, nota 34 en p. 276.

70 En concreto, por Paul Négulesco.

Ver Forteau, Mathias, "Le droit administratif global, signe d'une evolution des techniques du droit international?", en Bories, Clémentine (ed.), Un droit administratif global? A global administrative law?, Editions Pedone, París, 2012, pp. 178-180.

Ver también ibid., pp. 180-183.

71 Ver Dubin, "Le droit administratif global...," 2012, pp. 101-106.

Ver también Bories, "Histoire des phénomènes administratifs...", 2012, pp. 49-50.

72 Ver en general Weiler, Joseph, "GAL at a crossroads: preface to the symposium”, International Journal of Constitutional Law, 2015, 13, (2), pp. 463-464. 
ver todo tipo de problemas conceptuales, sin aclarar por qué el derecho internacional no ofrece una respuesta razonable. ${ }^{73}$

\section{Relación con otras iniciativas académicas recientes}

Una de las críticas más interesantes al GAL Project es la proveniente del proyecto sobre el Ejercicio del Poder Público Internacional del Instituto Max Planck de Heidelberg, a veces abreviado como IPA por su denominación en inglés de Exercise of International Public Authority. Este proyecto tuvo dos fases: una destinada a las instituciones internacionales en general, y otra a las instituciones judiciales en particular. ${ }^{74}$ El IPA Project se centró en el derecho público y se consideró a sí mismo como una síntesis de los intentos anteriores por estudiar y solucionar los vacíos normativos que produce la gobernanza global, dentro de los cuales incluyó al GAL Project. ${ }^{75}$ Sus autores definieron al ejercicio del poder público internacional como

73 Ver Casini, “Global administrative law...”, 2016, p. 563.

74 Ver Dann, Philipp \& Von Engelhardt, Marie, "Legal approaches to global governance and accountability: informal lawmaking, international public authority, and global administrative law compared", en Pauwelyn, Joost, Wessel, Ramses \& Wouters, Jan (eds.), Informal International Lawmaking, Oxford University Press, Oxford, 2012, p. 108.

Ver en general Von Bogdandy, Armin, Wolfrum, Rüdiger, Von Bernstorff, Jochen, Dann, Philipp \& Goldmann, Matthias (eds.), The exercise of public authority by international institutions: advancing international institutional law, Springer, Heidelberg, 2010; y Von Bogdandy, Armin \& Venzke, Ingo (eds.), International judicial lawmaking, Springer, Heidelberg, 2012.

Armin von Bogdandy lidera actualmente otra iniciativa que continúa su investigación comenzada en Heidelberg. Se trata del proyecto sobre el Ejercicio del Poder Público a Nivel Internacional, Supranacional y Doméstico de la Universidad de Fráncfort. Disponible en: http://www.normativeorders.net/en/research/projects-2012-2017/66-forschung/ forschungsprojekte-2012-2017/2210-the-exercise-of-public-authority-on-the-internationalsupranational-and-domestic-levels-reconstructing-public-law-to-face-the-challenges-ofmultilevel-governance, consulta del 3 de abril de 2017.

75 Ver Dann \& Von Engelhardt, "Legal approaches to global governance and accountability...", 2012, p. 108.

Ver también Von Bogdandy, Armin, Dann, Philipp \& Goldmann, Matthias, "Developing the publicness of public international law: towards a legal framework for global governance activities", German Law Journal, 2008, 9, (11), pp. 1375-1400, en pp. 1376-1395; y Von Bogdandy, Armin, Dann, Philipp \& Goldmann, Matthias, "Developing the publicness of public international law: towards a legal framework for global governance activities", en Von Bogdandy, Armin, Wolfrum, Rüdiger, Von Bernstorff, Jochen, Dann, Philipp \& Goldmann, Matthias (eds.), The exercise of public authority by international institutions: advancing international institutional law, Springer, Heidelberg, 2010, pp. 4-26. 
"todo tipo de actividad de gobernanza por instituciones internacionales, sean administrativas o intergubernamentales [que] determina a los individuos, asociaciones privadas, empresas, Estados, u otras instituciones públicos". ${ }^{76}$ La aproximación propuesta desde el Instituto Max Planck de Heidelberg pretendió dar una explicación más estrictamente conceptual de la internacionalización del derecho administrativo, que la ofrecida por el GAL Project. ${ }^{77}$ De ahí que Armin von Bogdandy y Matthias Goldmann, de la Universidad de Fráncfort, y Philipp Dann, de la Universidad Humboldt de Berlín, hayan destacado que las instituciones internacionales no pueden ser confundidas con las internas, como ocurre cada vez que se proponen soluciones normativas apoyadas en simples analogías domésticas. Precisamente por esa razón es que los enfoques del IPA y del GAL difieren. Según Von Bogdandy, Dann y Goldmann, la noción de GAL implica una fusión del derecho internacional y del derecho administrativo que no toma en consideración el hecho de que las normas jurídicas internacionales y las internas tienen una legitimidad de origen fundamentalmente distinta: el consentimiento estatal, por un lado, y la soberanía popular, por el otro. ${ }^{78}$ Kingsbury y Cassese han recogido escuetamente las críticas de este proyecto, al que el administrativista italiano ha llamado escuela de Heidelberg. ${ }^{79}$ Lo cierto es que al buscar instrumentos de derecho público interno para dilucidar asuntos no resueltos por el derecho internacional, por medio de un método descriptivo-prescriptivo, la iniciativa de esta escuela se superpone sustancialmente con la del GAL. ${ }^{80}$

76 Ver Von Bogdandy, Dann \& Goldmann, "Developing the publicness of public international law...”, 2008, p. 1376; y Von Bogdandy, Dann \& Goldmann, "Developing the publicness of public international law...", 2010, p. 5.

77 Ver Möllers, “Ten years of global administrative law”, 2015, p. 472.

Ver también Dann \& Von Engelhardt, "Legal approaches to global governance and accountability...”, 2012, pp. 108-113.

78 Ver Von Bogdandy, Dann \& Goldmann, "Developing the publicness of public international law...", 2008, p. 1393; y Von Bogdandy, Dann \& Goldmann, "Developing the publicness of public international law...”, 2010, pp. 24-25.

79 Ver Kingsbury, “The concept of 'law'...”, 2009, nota 2 en p. 24; y Cassese, “Global administrative law...", 2015, p. 465.

Ver también Dubin, "Le droit administratif global...", 2012, p. 98.

80 Ver Casini, “Global administrative law...”, 2016, p. 560; D’Aspremont, "Droit administratif global et droit international”, 2012, pp. 92-93; y Dubin, "Le droit administratif global...", 2012, p. 98. 
No debiera llamar la atención, por lo tanto, que la así denominada escuela de Heidelberg haya reconocido expresamente su conexión con el derecho institucional internacional, el derecho administrativo global y el constitucionalismo. ${ }^{81}$ Por su parte, el GAL Project también ha sido asociado con la constitucionalización del derecho internacional o global en más de una oportunidad. ${ }^{82}$ De hecho, las premisas de ambas visiones se acercan bastante. Tanto el GAL como el constitucionalismo surgen como una reacción a la pluralización de la gobernanza global y la marginalización del derecho internacional como instrumento de regulación. ${ }^{83}$ El constitucionalismo, en concreto, parte del supuesto de que los intereses de la comunidad internacional deben prevalecer sobre los derechos y obligaciones recíprocas de los Estados. Con este fin, y en contraposición al derecho de carácter privado o contractual que regula las relaciones bilaterales estatales, esta doctrina propone un derecho de naturaleza pública o constitucional que dé forma a dicho interés general comunitario. Autores alemanes o germano-parlantes vinculados a universidades de distintos países, como Alfred Verdross (1890-1980), Bruno Simma (Múnich), Bardo Fassbender (San Galo), Jan Klabbers (Helsinki), Anne Peters (Basilea) y Geir Ulfstein

81 Ver Von Bogdandy, Dann \& Goldmann, "Developing the publicness of public international law...”, 2008, pp. 1390-1395; y Von Bogdandy, Dann \& Goldmann, "Developing the publicness of public international law...", 2010, pp. 21-26.

El derecho institucional internacional estudia las organizaciones internacionales.

Ver en general Schermers, Henry \& Blokker, Niels, International institutional law, Martinus Nijhoff Publishers, Leiden, 2011; y Klabbers, Jan, An introduction to international organizations law, Cambridge University Press, Cambridge, 2015.

82 Ver e.g. Bories, "Histoire des phénomènes administratifs...", 2012, pp. 55-56; Mockle, "Le debat sur les principes et les fondements...”, 2012, pp. 17-22; y Möllers, “Ten years of global administrative law", 2015, p. 471.

Ver en general Kuo, Ming-Sung, "Between fragmentation and unity: the uneasy relationship between global administrative law and global constitutionalism", San Diego International Law Journal, 2009, 10, (2), pp. 439-467; y Kuo, Ming-Sung, "Taming governance with legality - Critical reflections upon global administrative law as small-C global constitutionalism", New York University Journal of International Law and Politics, 2011, 44, (1), pp. 55-102; y Savino, Mario, "What if global administrative law is a normative project?", International Journal of Constitutional Law, 2015, 13, (2), pp. 492-498.

83 Ver D’Aspremont, "Droit administratif global et droit international”, 2012, pp. 86-87. 
(Oslo), han tenido cierta preponderancia en esta doctrina. ${ }^{84}$ Sin embargo, no han sido los únicos en desarrollarla. ${ }^{85}$

La relación entre el GAL y el constitucionalismo admite más de un comentario. A modo de ejemplo, se pueden mencionar los de Alexander Somek y Mathias Forteau. Conforme con Somek, el derecho administrativo global vincula la descripción de diversos fenómenos con una agenda normativa limitada, destinada a establecer ciertos principios asociados a la preeminencia del derecho o rule of law y a la buena gobernanza o good governance. A grandes rasgos, estos objetivos no son muy distintos a los del constitucionalismo. No obstante, al ser de carácter pragmático, los fines del derecho administrativo global resultan considerablemente más modestos que aquellos buscados por cualquier forma de constitucionalización. ${ }^{86}$ Forteau ha agregado que la propuesta del GAL Project aparentemente permitiría liberarse de la distinción entre hard y soft law, dando lugar a un estudio del derecho internacional que pondría el acento en su administrativización o publicización. Sin embargo, esta perspectiva no es nueva. En gran medida, ya ha sido planteada por las concepciones constitucionalistas o constitucionalizantes del derecho internacional. ${ }^{87} \mathrm{El}$ problema que Forteau ve en esta línea de pensamiento es que parte de la base de que la naturaleza del derecho internacional ha evolucionado desde el derecho privado al derecho público, cuando lo cierto es que actualmente se puede hablar con propiedad tanto de una privatización del derecho internacional, como de una constitucionalización de este. ${ }^{88}$

¿Qué han dicho los principales autores del GAL Project sobre el derecho administrativo global y el derecho constitucional internacional o global? Nico Krisch ha concordado, de alguna manera, con lo señalado

\footnotetext{
84 Ver e. g. Verdross, Alfred, Der verfassung der völkerrechtsgemeinschaft, Springer-Verlag, Berlín, 1926; Simma, Bruno, "From bilateralism to community interest in international law”, Recueil des Cours, 1994/VI, (250), pp. 217-384; Fassbender, Bardo, The United Nations Charter as the constitution of the international community, Martinus Nijhoff Publishers, Leiden, 2009; y Klabbers, Jan, Peters, Anne \& Ulfstein, Geir, The constitutionalization of international law, Oxford University Press, Oxford, 2011.

85 Ver Neff, Justice among nations..., 2014, pp. 453-458.

86 Ver Somek, Alexander, “The concept of 'law'...”, 2010, p. 986; y Somek, "Administration without sovereignty", 2010, p. 272.

87 Ver Forteau, “Le droit administratif global...”, 2012, pp. 170-171.

88 Ver ibid., pp. 172-174.

Ver también ibid., pp. 174-183.
} 
por Somek. En su opinión, tanto el constitucionalismo como el GAL son intentos por enfrentarse al déficit democrático propio de la gobernanza global. ${ }^{89}$ No obstante, mientras el primero tiene una visión holística, el segundo posee un alcance más modesto, a menor escala, que lo hace más adecuado para el estudio académico y la reforma política. ${ }^{90}$ Krisch también ha señalado, junto a Benedict Kingsbury y Richard Stewart, que el GAL admite dos tipos de aproximaciones: desde abajo (bottom-up) y desde arriba (top-down), según los principios normativos se obtengan del derecho administrativo doméstico o comparado, o bien provengan de una red de tratados o de decisiones adoptadas a nivel supraestatal, respectivamente. ${ }^{91}$ Aunque posible, la segunda de estas alternativas no sería recomendable en el GAL, principalmente por dos razones: primero, requiere una institucionalización de regímenes administrativos informales actualmente efectivos; y, segundo, adolece de una insoslayable falta de legitimidad democrática. ${ }^{92}$ Stewart ha destacado esta diferencia con el constitucionalismo, que opera precisamente desde arriba. ${ }^{93}$ Por último, Sabino Cassese ha indicado que el derecho administrativo global no cuenta con una estructura constitucional sobre la cual erigirse, como la que tiene el derecho doméstico. Si bien el autor italiano acepta que hay ciertos indicios de un proceso de constitucionalización en el derecho internacional, como, por ejemplo, en el ámbito de los derechos humanos, este es todavía rudimentario y no se asemeja a las reglas y principios domésticos. Para Cassese, es justamente la ausencia de esta base constitucional lo que provocaría los problemas de accountability en el espacio global. ${ }^{94}$

89 Ver Krisch, "Global administrative law and the constitutional ambition", 2010, pp. 246-251.

90 Ver ibid, pp. 245-246 y 251-266.

91 Ver Kingsbury, Krisch \& Stewart, "The emergence of global administrative law", 2005, pp. 52-59; Stewart, "U.S. administrative law...", 2005, pp. 71-73 y 76-107; y Stewart, "The global regulatory challenge...”, 2005, pp. 709-712 y 753-759.

Ver también Kingsbury \& Donaldson, “Global administrative law”, 2015, párrafo 24.

92 Ver Kingsbury, Krisch \& Stewart, "The emergence of global administrative law”, 2005, p. 57. Ver también Stewart, “U.S. administrative law...", 2005, pp. 88 y 106-107.

93 Ver Stewart, “The normative dimensions and performance...”, 2015, p. 506.

94 Ver Cassese, “Administrative law without the state...", 2005, pp. 687-689.

Ver también Cassese, The global polity..., 2012, pp. 63-69; Cassese, "Global administrative law...", 2015, pp. 465-466; y Kingsbury \& Donaldson, "Global administrative law”, 2015, párrafo 57. 
Otra iniciativa que parte de premisas muy similares a las del Instituto Max Planck de Heidelberg y del GAL Project, es el proyecto sobre la Creación Jurídica Informal Internacional, abreviado como IN-LAW por sus autores en razón de su nombre en inglés de Informal International Lawmaking. ${ }^{95}$ Este proyecto fue desarrollado bajo los auspicios del Instituto de La Haya para la Internacionalización del Derecho y el liderazgo de Joost Pauwelyn, del Instituto de Graduados de Ginebra; Ramses Wessel, de la Universidad de Twente; y Jan Wouters, de la Universidad Católica de Lovaina. El INLAW prescinde de ciertas formalidades usualmente asociadas a la creación jurídica internacional tradicional, lo cual puede observarse desde el punto de vista del resultado, del proceso y de los actores involucrados en la cooperación transfronteriza. Así, esta puede ser informal en el sentido de que: primero, no conduce a una fuente tradicional del derecho internacional, como un tratado, sino que a guías, estándares, declaraciones, etc., de carácter normativo, en tanto dirigen o afectan la conducta de los distintos actores (resultado); segundo, tiene lugar en redes o foros de contornos imprecisos, más que en organizaciones internacionales convencionales, lo que no impide que puedan existir reglas procesales detalladas, un personal permanente o una sede física, pero sí excluye a las simples negociaciones (proceso); y, tercero, no involucra a actores diplomáticos tradicionales, como jefes de Estado, ministros de relaciones exteriores o embajadores, sino que a otros ministerios, reguladores domésticos, agencias, entidades subfederales, a otros poderes estatales, como el legislativo o judicial, e incluso a actores privados junto a los públicos (actores). ${ }^{96}$

Como la escuela de Heidelberg y el GAL Project, el proyecto sobre el IN-LAW analizó la creación jurídica más allá de las fuentes y sujetos tra-

95 Ver en general Pauwelyn, Joost, Wessel, Ramses \& Wouters, Jan (eds.), Informal international lawmaking, Oxford University Press, Oxford, 2012; y Pauwelyn, Joost, Wessel, Ramses \& Wouters, Jan, "When structures become shackles: stagnation and dynamics in international lawmaking”, European Journal of International Law, 2014, 25, (3), pp. 733-763.

Ver también Berman, Ayelet, Duquet, Sanderijn, Pauwelyn, Joost, Wessel, Ramses \& Wouters, Jan (eds.), Informal international lawmaking: case studies, Torkel Opsahl Academic EPublisher, The Hague, 2012.

En la página de internet del Instituto de La Haya para la Internacionalización del Derecho se pueden ver más detalles de este proyecto. Disponible en http://www.hiil.org/ project/informal-international-law-making-and-accountability

96 Ver Pauwelyn, Joost, "Informal international lawmaking: framing the concept and research questions”, en Pauwelyn, Joost, Wessel, Ramses \& Wouters, Jan (eds.), Informal international lawmaking, Oxford University Press, Oxford, 2012, pp. 15-31. 
dicionales del derecho internacional, y se preguntó por los problemas de accountability que este fenómeno conlleva. Y como estos otros proyectos, el IN-LAw Project no se quedó en la sola descripción, sino que tuvo también un carácter normativo. ${ }^{97}$ La vinculación entre las tres iniciativas es evidente. Por ejemplo, el proyecto del Instituto de La Haya expresamente utilizó la definición de ejercicio del poder público internacional del IPA Project. ${ }^{98}$ Así mismo, al igual que en el GAL, en el proyecto del Instituto de La Haya también se debatió sobre la naturaleza jurídica de la noción sobre la cual descansa. En este caso, del IN-LAW. ${ }^{99}$ No obstante, esta iniciativa se diferenció en algunos aspectos de la escuela de Heidelberg, así como también del GAL. En primer lugar, el IN-LAW solo estudió la creación jurídica informal, en tanto que el GAL y el IPA analizan esta y la creación jurídica formal. ${ }^{100}$ De hecho, el IN-LAW y el GAL se distinguen de otras iniciativas académicas por sus respectivos campos de estudio: la creación jurídica informal internacional, en el primero, y el derecho administrativo global, en el segundo. Sin embargo, como bien destacaron sus autores, no todo el IN-LAW tiene carácter administrativo. ${ }^{101}$ Segundo, mientras el proyecto de la NYU incluye a la cooperación meramente privada, el del Instituto de La Haya para la Internacionalización del Derecho la excluyó, y el del Instituto Max Planck de Heidelberg solo la comprende en la medida que los actores particulares ejerzan poder público. ${ }^{102} \mathrm{Al}$ respecto, José Álvarez ha

97 Ver e.g. Dann \& Von Engelhardt, "Legal approaches to global governance and accountability...", 2012.

98 Ver Pauwelyn, "Informal international lawmaking...", 2012, pp. 21 y 28.

Ver también Von Bogdandy, Dann \& Goldmann, "Developing the publicness of public international law...”, 2008, p. 1376; y Von Bogdandy, Dann \& Goldmann, "Developing the publicness of public international law...", 2010, p. 5.

99 Ver e.g. las contribuciones de Joost Pauwelyn, Dick Ruiter y Ramses Wessel, Jean d'Aspremont y Andrea Bianchi en Pauwelyn, Wessel \& Wouters (eds.), Informal international lawmaking, Oxford University Press, Oxford, 2012, pp. 125-215.

100 Ver Dann \& Von Engelhardt, "Legal approaches to global governance and accountability...", 2012, p. 114.

Ver también Pauwelyn, "Informal international lawmaking...", 2012, p. 31.

101 Ver Pauwelyn, “Informal international lawmaking...,", 2012, p. 31.

Ver también Pauwelyn, Wessel \& Wouters, "When structures become shackles...", 2014, pp. 742-744.

102 Ver Dann \& Von Engelhardt, "Legal approaches to global governance and accountability...", 2012, p. 113-115.

Ver también Pauwelyn, "Informal international lawmaking...", 2012, pp. 19 y 21. 
indicado que el proyecto sobre el IPA se limitó a analizar entidades formales e informales donde el Estado retiene algún rol, dejando de lado entidades como las organizaciones no gubernamentales o las agrupaciones de empresas multinacionales. ${ }^{103}$

\section{Nueva York y New Haven}

Como bien ha destacado Jean d'Aspremont, el ejercicio del poder estatal se ha vuelto más difuso en la sociedad poswestfaliana. ${ }^{104}$ Las teorías del derecho administrativo global, de la escuela de Heidelberg, del constitucionalismo y de la creación jurídica informal internacional, así como del derecho transnacional, analizan este fenómeno, promoviendo trasplantes legales y el cruce de límites entre sistemas o regímenes jurídicos. ${ }^{105}$ Respecto al derecho global, las distintas doctrinas que han especulado sobre este han intentado explicar dicha realidad, partiendo de una constatación compartida: la superación del Estado como actor cuasiexclusivo de las relaciones internacionales y, por lo tanto, del derecho internacional como orden normativo eminentemente interestatal. ${ }^{106}$ Es desde este punto de vista que el GAL Project se relaciona con el cosmopolitismo y los derechos transnacional, mundial y global a secas, por un lado, y con otras escuelas que han estudiado la sociedad internacional y su derecho, generalmente conocidas por el lugar donde se encuentra la universidad de la mayoría de sus cultores, por otro. En efecto, el derecho administrativo global se vincula tanto con los postulados de Von Stein, Reinsch y Potter, como con los de Clyde Eagleton (1891-1958) y Thomas Franck (1931-2009) de la NYU. Junto a Wolfgang Friedmann (1907-1972) de la Universidad de Columbia, Eagleton y Franck dieron lugar a la escuela de Manhattan.

\footnotetext{
103 Ver Álvarez, José, “Beware: Boundary crossings’. A critical appraisal of public law approaches to international investment law", Journal of World Investment $\lesssim$ Trade, 2016, 17, (2), pp. 171-228, en pp. 184-185.

104 Ver D’Aspremont, "Droit administratif global et droit international”, 2012, p. 85.

Ver también D'Aspremont, Jean, "Cognitive conflicts and the making of international law: from empirical concord to conceptual discord in legal scholarship", Vanderbilt Journal of Transnational Law, 2013, 46, (4), pp. 1119-1147.

105 Ver Casini, “Global administrative law...”, 2016, pp. 548-549; y Álvarez, “Beware: Boundary crossings'...”, 2016, pp. 181-191.

106 Ver Hennebel, "Penser le droit administratif global...", 2012, p. 73.
} 
Una de las principales preocupaciones de Franck fue, precisamente, el constitucionalismo. ${ }^{107}$ La conexión del GAL con los postulados de este y con los de la escuela de Manhattan obedece a que el derecho administrativo global no es otra cosa que una forma de solidarismo, en sus dos variantes: como aproximación tecnocrática y sociológica al derecho. La idea central del solidarismo, en ambas vertientes, es la interdependencia. Esto explica que busque reemplazar la sociedad internacional centrada en el Estado soberano y la independencia por una comunidad internacional donde se promueva la cooperación y el bienestar general de todas las personas. Al menos en sus orígenes, autores franceses o franco-parlantes como Léon Duguit (1859-1928) de la Universidad de Burdeos, y Louis Renault (18431918), Alejandro Álvarez (1868-1960) y Georges Scelle de la Universidad de París, predominaron en el solidarismo. ${ }^{108}$ Con el tiempo, su influencia se extendió a otros países y continentes. Es por esta razón que el GAL Project se vincula tanto con el constitucionalismo y la escuela de Manhattan, como con otro proyecto académico con aspiraciones de políticas públicas o public policy — la escuela de New Haven—. ${ }^{109}$ Esta es una corriente doctrinal que surgió en la segunda mitad del siglo Xx como una reacción al positivismo y realismo jurídicos entonces imperantes, que en su análisis del proceso legislativo y judicial, respectivamente, dejaban de lado el estudio de los aspectos extrajurídicos del derecho. ${ }^{110}$ También conocida como escuela

107 Ver Neff, Justice among nations..., 2014, pp. 427, 452-453 y 458.

Ver en general Kingsbury, Benedict, "Neo-madisonian global constitutionalism: Thomas M. Franck's democratic cosmopolitan prospectus for managing diversity and world order in the twenty-first century", New York University Journal of International Law and Politics, 2003, 35, (2), pp. 291-301.

Excepcionalmente, Jean d'Aspremont ha equiparado el GAL Project con la escuela de Manhattan.

Ver D’Aspremont, "Droit administratif global et droit international", 2012, pp. 83-84.

108 Ver Neff, Justice among nations..., 2014, pp. 285-297, 308-310, 373-378 y 424-425.

Ver también Koskenniemi, Martti, The gentle civilizer of nations. The rise and fall of international law 1870-1960, Cambridge University Press, Cambridge, 2005, pp. 266-352.

109 Ver Neff, Justice among nations..., 2014, pp. 373-378, 424-432 y 452-461.

Ver también Dubin, "Le droit administratif global...", 2012, pp. 106-113.

Cf. Orakhelashvili, Alexander, "International law, international politics and ideology", en Orakhelashvili, Alexander (ed.), Research handbook on the theory and history of international law, Edward Elgar Publishing, Cheltenham, 2011, pp. 335-339.

110 Ver Berman, Paul Schiff, "A pluralist approach to international law", Yale Journal of International Law, 2007, 32, (2), pp. 301-329, en pp. 301-302; Koh, Harold Hogjou, "Is there 
de Yale, su nombre hace referencia al lugar geográfico donde comenzó, tal como antes lo hiciera el de escuela de Viena. ${ }^{111}$ Harold Lasswell (19021978), un cientista político, y Myres McDougal (1906-1998), un abogado, dieron inicio a la escuela de New Haven, que más tarde pasaría a estar integrada por otros profesores, como Richard Falk, Rosalyn Higgins, John Norton Moore, Michael Reisman, Oscar Schachter y Burns Weston, no necesariamente vinculados a la Universidad Yale. ${ }^{112}$ Como el GAL Project,

a 'new' New Haven School of International Law?", Yale Journal of International Law, 2007, 32, (2), pp. 559-573, en pp. 561-562; y Wiessner, Siegfried, “The New Haven School of Jurisprudence: a universal toolkit for understanding and shaping the law", Asia Pacific Law Review, 2010, 18, (1), pp. 45-61, en pp. 46-47.

Ver también Suzuki, Eisuke, “The New Haven School of International Law: an invitation to a policy-oriented jurisprudence", Yale Studies in World Public Order, 1974, (1), pp. 1-48, en p. 6; Falk, Richard, "Casting the spell: the New Haven School of International Law”, Yale Law Journal, 1995, 104, (7), pp. 1991-2008, en pp. 1991-1192; y Reisman, Michael, "Theory about law: jurisprudence for a free society", Yale Law Journal, 1999, 108, (5), pp. 935-939, en pp. 935-938.

111 Ver Suzuki, “The New Haven School...”, 1974, p. 5.

Ver Hathaway, Oona, "The continuing influence of the New Haven School", Yale Journal of International Law, 2007, 32, (2), pp. 553-558, en pp. 553-555; y Koh, "Is there a 'new' New Haven School...", 2007, p. 559.

La escuela de Viena estuvo integrada por Hans Kelsen y sus discípulos Josef Kunz (1890-1970) y Alfred Verdross, profesores de la universidad que lleva el nombre de la capital austriaca. El positivismo de esta escuela es célebre por su normativismo, eminentemente formalista.

Ver Neff, Justice among nations..., 2014, pp. 367-371, 374, 424, 429, 453 y 456.

Verdross, sin embargo, era naturalista. En su opinión, la grundnorm o norma fundamental de la pirámide kelseniana consiste en una idea o sentido general de justicia. Desde este punto de vista, su noción de derecho natural era esencialmente organicista y no trascendente, pues descansaba en una especie de conciencia interna colectiva de la comunidad internacional.

Ver ibid., pp. 379-380.

Sobre la escuela de Viena, ver en general Kunz, Josef, "The 'Vienna School' and international law", New York University Law Quarterly Review, 1934, 11, (3), pp. 370-421; y Lador-Lederer, Josef, "Some observations on the 'Vienna School' in International Law", Netherlands International Law Review, 1970, 17, (2), pp. 126-150.

112 Ver Hathaway, "The continuing influence...", 2007, pp. 553-555; Koh, "Is there a 'new' New Haven School...”, 2007, pp. 559-561; y Neff, Justice among nations..., 2014, pp. 377-378 y 428-429.

De los autores mencionados, solo Reisman ha trabajado en Yale. Falk es profesor de la Universidad de Princeton, mientras que Higgins y Weston lo fueron de la London 
la escuela de New Haven ha sido prolífica. ${ }^{113}$ Su texto fundamental es el tratado en dos volúmenes que Lasswell y McDougal publicaron en 1992 bajo el título de Jurisprudence for a free society. ${ }^{114}$

Para la escuela de Yale, el derecho internacional no es solo un conjunto de reglas, sino que constituye un proceso social de adopción o toma de decisiones autoritativas. ${ }^{115}$ Cada proceso debe ser continuo, ya que las simples elecciones ocasionales quedan excluidas del análisis de esta escuela. Así mismo, el poder debe ser efectivamente ejercido en el proceso respectivo, en el sentido de que las decisiones no solo sean adoptadas, sino también ejecutadas. ${ }^{116}$ Esto significa que el derecho no proviene únicamente de poderes estatales, como el ejecutivo, legislativo o judicial, sino que emana de ciertas comunicaciones de grupos políticamente relevantes que crean la expectativa de un comportamiento futuro apropiado, en la medida que dichas comunicaciones tengan un contenido prescriptivo y provengan de autoridades a las cuales la comunidad correspondiente les reconozca un poder de esta naturaleza. ${ }^{117} \mathrm{El}$ contexto del problema por

School of Economics and Politicial Science (LSE) de la Universidad de Londres, y de la Universidad de Iowa.

Excepcionalmente, algunos miembros de esta escuela ni siquiera estudiaron en la Universidad Yale. Es el caso de los profesores John Norton Moore, exalumno de la Universidad de Virginia, y Oscar Schachter, de la Universidad de Columbia.

113 Ver e.g., Wiessner, Siegfried \& Willard, Andrew, "Policy-oriented jurisprudence”, German Yearbook of International Law, 2001, (44), pp. 96-112, nota 1 en p. 96; Berman, "A pluralist approach to international law", 2007, nota 18 en p. 305; y Reisman, Michael, Wiessner, Siegfried \& Willard, Andrew, "New Haven School: a brief introduction”, Yale Journal of International Law, 2007, 32, (2), pp. 575-582, nota 2 en p. 575.

114 Ver Lasswell, Harold \& McDougal, Myres, Jurisprudence for a free society: studies in law, science and policy, Martinus Nijhoff Publishers-New Haven Press, Dordrecht-New Haven, 1992.

Ver también Falk, "Casting the spell...", 1995; y Reisman, “Theory about law...”, 1999.

115 Ver Koh, “Is there a 'new' New Haven School...”, 2007, pp. 562-563.

Ver también Wiessner, “The New Haven School...”, 2010, p. 47.

116 Ver Reisman, Wiessner \& Willard, “New Haven School...”, 2007, pp. 579-580.

Ver también Reisman, Michael, "The view from the New Haven School of International Law", American Society of International Law Proceedings, 1992, (86), pp. 118-125, en p. 121.

117 Ver Wiessner \& Willard, "Policy-oriented jurisprudence", 2001, pp. 101 y 107-108; Berman, "A pluralist approach to international law", 2007, pp. 305-307; y Wiessner, "The New Haven School...", 2010, pp. 47-50.

Ver también Reisman, Michael, "International lawmaking: a process of communication", 
estudiar y la perspectiva desde la cual se le analiza juegan un rol central en la determinación del carácter jurídico de las decisiones respectivas. ${ }^{118}$ Según esta escuela, los procesos de adopción de decisiones autoritativas deben promover valores normativos, destinados a establecer un orden público en el que prevalezca la dignidad humana. ${ }^{119}$ Aun cuando parte de la observación, la escuela de New Haven se caracteriza por ser innegablemente activista y tener un top-down approach, al proponer soluciones desde arriba, en este caso provenientes de la academia. ${ }^{120}$ Desde esta perspectiva, el derecho y las políticas públicas quedan estrechamente vinculados, al punto de que las reglas de derecho internacional deben reflejar las necesidades de la política exterior, y viceversa. ${ }^{121}$ No por nada, los otros nombres que ha recibido la escuela de Yale son policy-oriented jurisprudence y law, science and policy, que vagamente se pueden traducir al castellano como 'teoría orientada a las políticas públicas' o ‘derecho, ciencia y políticas públicas'. De hecho, estas fueron las denominaciones originales que recibió, puesto que el nombre de escuela de New Haven recién comenzó a ser utilizado en la década de los sesenta. ${ }^{122}$ No es una simple coincidencia que la escuela de Yale haya sido una de las primeras en reconocer la importancia del derecho transnacional de Philip Jessup, quien a su vez manifestó su reconocimiento hacia el trabajo de McDougal. ${ }^{123}$

American Society of International Law Proceedings, 1981, (75), pp. 101-120, en pp. 105-119; y Reisman, "The view from the New Haven School...", 1992, pp. 121-123.

118 Ver Wiessner \& Willard, "Policy-oriented jurisprudence”, 2001, pp. 102-107.

Ver también Reisman, "The view from the New Haven School...”, 1992, p. 120; Reisman, "Theory about law...", 1999, pp. 938-939; y Reisman, Wiessner \& Willard, "New Haven School...”, 2007, pp. 576-580.

119 Ver Koh, "Is there a 'new' New Haven School...", 2007, p. 563; y Wiessner, "The New Haven School...”, 2010, pp. 48 y 50-51.

Ver también Reisman, “Theory about law...", 1999, p. 939; y Reisman, Wiessner \& Willard, "New Haven School...", 2007, pp. 575-577 y 580.

120 Ver Neff, Justice among nations..., 2014, pp. 429-430.

121 Ver Koh, “Is there a 'new' New Haven School...”, 2007, p. 563.

122 Fue Richard Falk quien empezó a usar esta denominación por esos años.

Ver Chen, Lung-chu, "Perspectives from the New Haven School", American Society of International Law Proceedings, 1993, (87), pp. 407-411, en p. 408; Hathaway, "The continuing influence...", 2007, p. 554; y Reisman, Wiessner \& Willard, "New Haven School...”, 2007, nota 2 en p. 575 y 577.

123 Ver Koh, "Is there a 'new' New Haven School...", 2007, p. 564.

Ver también Jessup, “Diversity and uniformity...”, 1964, p. 352. 
Al igual que otras escuelas y aproximaciones teóricas, la escuela de New Haven ha sido criticada en doctrina. En este caso, por fusionar el derecho y las políticas públicas de tal manera, que corre el riesgo de convertirse en un intento de transformar al derecho internacional en un mero instrumento de política exterior, como ocurre en los Estados totalitarios o en la versión más radical de la realpolitik. ${ }^{124}$ Por este motivo, Oscar Schachter y Richard Falk se alejaron de la escuela de Yale durante la guerra de Vietnam (1955-1975), en rechazo a la tendencia de McDougal y otros de aplicar la teoría de una manera selectiva, a fin de favorecer los intereses de política exterior de los Estados Unidos. ${ }^{125}$ La escuela de New Haven también ha sido cuestionada por su excesiva utilización del argot o jerga técnico-académica en sus escritos, por su decidido carácter elitista y por su escaso impacto más allá de las aulas universitarias estadounidenses. ${ }^{126}$ Cabe destacar que la aproximación de la escuela de Yale no es nueva en el derecho. Por un lado, la sociología y antropología jurídicas vienen estudiando desde comienzos del siglo xx los procesos sociales y las estructuras de autoridad y control, respectivamente. Por otro, la idea del dinamismo en el derecho, como resultado de conflictos, fue propuesta por la escuela alemana de la jurisprudencia de intereses en el siglo XIX, y el rechazo de la reducción de la toma de decisiones a un simple silogismo neutro, consistente en encontrar las normas aplicables a los hechos relevantes, fue planteado por el realismo jurídico estadounidense en el siglo xx. ${ }^{127}$ Sin perjuicio de lo anterior, la escuela de New Haven ha sido de gran importancia para dilucidar algunos de los problemas que son propios del derecho internacional contemporáneo, y sus postulados han incentivado directa o indirectamente a escuelas y teorías posteriores, como la de Manhattan, la de Heidelberg, la del IN-LAW y la del GAL. No es una casualidad, por lo tanto, que todas estas aproximaciones tengan un aspecto descriptivo y

\footnotetext{
124 Ver Suzuki, “The New Haven School...”, 1974, p. 7.

125 Ver Koh, "Is there a 'new' New Haven School...", 2007, p. 563; Orakhelashvili, “International law, international politics and ideology”, 2011, pp. 335-339; y D'Aspremont, "Cognitive conflicts and the making of international law...", 2013, pp. 1119-1147.

Ver e.g. Falk, "Casting the spell...", 1995, pp. 1996-2007.

126 Ver Neff, Justice among nations..., 2014, p. 431.

Ver también ibid., pp. 428-430.

Cf. Suzuki, “The New Haven School...”, 1974, p. 8 y nota 22 en pp. 10-11.

127 Ver Wiessner \& Willard, "Policy-oriented jurisprudence”, 2001, p. 97.

Ver también ibid., pp. 98-100.
} 
otro prescriptivo, al igual que el constitucionalismo. ${ }^{128}$ Por ser corrientes de pensamiento solidarista, dichas escuelas y proyectos se asemejan en más de una faceta a lo planteado por el cosmopolitismo, que a su vez se vincula con la noción de derecho transnacional. ${ }^{129}$

Ilustra bien esta relación la teoría del proceso jurídico trasnacional de Harold Koh, definido por este como la interacción institucional por medio de la cual las normas globales no solo son creadas e interpretadas, sino que internalizadas en los distintos sistemas jurídicos domésticos. ${ }^{130}$ Discípulo de Reisman y también profesor de Yale, Koh ha combinado las propuestas de Lasswell y McDougal con las de Robert Cover (1943-1986), otro profesor de la misma universidad. Cover consideraba que la noción de derecho de John Austin (1790-1859) era insuficiente para describir los fenómenos jurídicos. En su opinión, el derecho no está únicamente compuesto de mandatos generales del soberano acompañados de la amenaza de sanciones, sino que se construye a partir de diversas comunidades generadoras de normas, que compiten entre sí. ${ }^{131}$ Koh ha rescatado esta noción de Cover, para articularla con la concepción jurídica de Lasswell y McDougal, del derecho como proceso social de toma de decisiones

128 Para la escuela de Yale, ver Wiessner \& Willard, "Policy-oriented jurisprudence”, 2001, pp. 96-97 y 100-101; Koh, "Is there a 'new' New Haven School...”, 2007, pp. 569-570; y Reisman, Wiessner \& Willard, “New Haven School...”, 2007, pp. 575-576.

129 Ver Sato, “Towards the institutionalization of...”, 2009, pp. 1142-1144.

Ver también Guilmain, "Du droit cosmopolitique au droit global...", 2014, pp. 230236; Neff, Justice among nations..., 2014, pp. 285-297, 308-310, 373-378, 424-432 y 452-461; y Álvarez, “'Beware: Boundary crossings'...”, 2016, pp. 181-186.

130 Ver Koh, Harold Hongjou, "Transnational legal process”, Nebraska Law Review, 1996, 75, (1), pp. 181-207, en pp. 183-191 y 203-207; Koh, Harold Hongjou, "Why do nations obey international law?", Yale Law Journal, 1997, 106, (8), pp. 2599-2659, en pp. 2602, 2646 y 2655-2658; Koh, Harold Hongjou, "Bringing international law home", Houston Law Review, 1998, 35, (3), pp. 623-681, en pp. 626-655 y 674-679; Koh, Harold Hongjou, "How is international human rights law enforced?", Indiana Law Journal, 1999, 74, (4), pp. 1397-1417, en pp. 1399-1400 y 1408-1416; Koh, "Is there a 'new' New Haven School...", 2007, pp. 566-569; y Koh, “Why transnational law matters”, 2006, en pp. 746-747.

131 Ver en general Cover, Robert, "The Supreme Court, 1982 term - Foreword: nomos and narrative", Harvard Law Review, 1983, 97, (1), pp. 4-68; Cover, Robert, "The uses of jurisdictional redundancy: interest, ideology, and innovation", William and Mary Law Review, 1981, 22, (4), pp. 639-682; y Cover, Robert, “The folktales of justice: tales of jurisdiction”, Capital University Law Review, 1985, 14, (2), pp. 179-203.

Ver en general Austin, John, The province of jurisprudence determined, John Murray, London, 1832. 
autoritativas. De esta manera, la propuesta de Koh no solo constituye un intento de recuperar la noción de derecho transnacional de Jessup, sino también de actualizarla a las necesidades disciplinarias actuales. ${ }^{132}$

\section{Conclusión}

Académicos de distintas nacionalidades han planteado denominaciones alternativas al derecho internacional, principalmente desde universidades de Europa occidental y Norteamérica. De estos conceptos, los de mayor influencia probablemente han sido el cosmopolitismo de Immanuel Kant y el derecho transnacional de Philip Jessup. Excepcionalmente, se han formado escuelas de pensamiento en torno a alguna de las nociones propuestas. Vale decir, agrupaciones de autores ligados, de una u otra manera, a una o más instituciones que trabajan diversos temas a partir de determinadas premisas. Este, sin duda, es el caso de la escuela de New Haven y del GAL Project. Algunas de estas iniciativas académicas tienen una naturaleza eminentemente descriptiva, como el derecho transnacional, el derecho mundial y el nuevo ius gentium, mientras que otras se caracterizan por ser descriptivo-prescriptivas, como el cosmopolitismo, la escuela de New Haven, el constitucionalismo, el nuevo derecho global, la escuela de Heidelberg, el IN-LAW Project y el GAL. Así mismo, las alternativas conceptuales sugeridas pueden tener una aproximación exclusivamente desde arriba (top-down approach), como la escuela de New Haven, el derecho mundial, el constitucionalismo, el nuevo derecho global y el nuevo ius gentium; o una combinación de acercamientos desde arriba y desde abajo (bottom-up), como los derechos cosmopolita y transnacional, la escuela de Heidelberg, el IN-LAW Project y el GAL.

132 Ver Berman, “A pluralist approach to international law”, 2007, pp. 302-311 y 328-329.

Paul Berman también ha formulado su propia aproximación teórica para superar las falencias del derecho internacional. Denominada "derecho y globalización", recoge los postulados del pluralismo jurídico. Sin embargo, no se aleja mucho de lo antes expuesto por Jessup, Lasswell, McDougal, Cover y Koh.

Ver ibid.; y Berman, Paul Schiff, "From international law to law and globalization", Columbia Journal of Transnational Law, 2005, 43, (2), pp. 485-556.

Según Michael Reisman, Siegfried Wiessner y Andrew Willard, la teoría de Berman solo se queda en la descripción del problema, sin plantear soluciones a este.

Ver Reisman, Wiessner \& Willard, “New Haven School...”, 2007, pp. 580-582. 
No hay duda de que el GaL Project ha sido exitoso. Así lo atestiguan los innumerables escritos que se refieren al derecho administrativo global, tengan o no que ver realmente con el tema. Sin embargo, la buena aceptación que ha tenido el GAL no significa que sus postulados sean del todo originales. Benedict Kingsbury ha admitido esto, al indicar que el derecho administrativo global no propone una idea inédita, pero le asigna a esta significados novedosos que renuevan su importancia. ${ }^{133}$ Las premisas del GAL lo ubican como una corriente más del solidarismo, tendencia que agrupa en su seno tanto al transnacionalismo y las escuelas de New Haven, Manhattan y Heidelberg, como al constitucionalismo y la creación jurídica informal internacional. Por esta razón, Lorenzo Casini ha observado correctamente que el derecho administrativo global solo podrá prosperar como área de estudio si empieza a trabajar junto a otras aproximaciones y escuelas. De lo contrario, solo será un nombre nuevo para un fenómeno viejo. ${ }^{134}$ Efectivamente, el derecho administrativo global ganará en permanencia cuando sea debidamente contextualizado, y se lo relacione con otros acercamientos a un problema que ha desvelado a muchos: el del derecho más allá del Estado. Lo cierto es que cuesta no coincidir con aquellos que critican la noción de derecho internacional, por no reflejar las complejidades de un mundo globalizado hasta hace poco desconocido, no por la novedad intrínseca de eso que los franceses llaman mundialización, sino por la escala y profundidad que este fenómeno ha alcanzado.

Nuevos sujetos del derecho requieren de nuevas fuentes jurídicas que los regulen. Es lo que han planteado las distintas corrientes de solidarismo mencionadas, dentro de las cuales se encuentra el GAL Project. El afán descriptivo y prescriptivo de este proyecto es loable, a pesar de que únicamente el primero de dichos objetivos tenga reales posibilidades de llegar a puerto. Como bien ha observado Stephen Neff respecto de la escuela de New Haven y del constitucionalismo, quizá la impronta de estos consista en abrir las mentes, más que en obtener resultados específicos. ${ }^{135}$ Lo mismo puede decirse de la escuela de Heidelberg y del proyecto sobre el IN-LAW, así como del GAL Project. Y eso, ciertamente, no es poco.

\footnotetext{
133 Kingsbury, "Weighing global regulatory rules and decisions...”, 2009, pp. 91-92.

134 Ver Casini, "Beyond drip-painting?...”, 2015, p. 477.

135 Ver Neff, Justice among nations..., 2014, p. 458.
} 


\section{Referencias}

Al Hajjaji, Shams Al Din, "Review of The New Global Law by Rafael Domingo", Berkeley Journal of International Law, 2014, 32, (1), pp. 268-280.

Álvarez, José, “'Beware: Boundary crossings'. A critical appraisal of public law approaches to international investment law", Journal of World Investment \& Trade, 2016, 17, (2), pp. 171-228.

Auby, Jean-Bernard, La globalisation, le droit et l'état, Éditions Montchrestien, París, 2003.

Austin, John, The province of jurisprudence determined, John Murray, London, 1832.

Bederman, David, "World law transcendent", Emory Law Journal, 2005, 54, (5), pp. 53-77.

Bentham, Jeremy, An introduction to the principles of morals and legislation, T. Payne and Son, London, 1780.

Benvenisti, Eyal, The law of global governance, Brill-Nijhoff, Leiden, 2014.

Berman, Ayelet, Duquet, Sanderijn, Pauwelyn, Joost, Wessel, Ramses \& Wouters, Jan (eds.), Informal international lawmaking: case studies, Torkel Opsahl Academic EPublisher, The Hague, 2012.

Berman, Harold, "Introduction to the World Law Institute", Emory International Law Review, 2008, 22, (1), pp. 1-6.

Berman, Harold, "The historical foundations of law", Emory Law Journal, 2005, 54, (5), pp. 13-24.

Berman, Harold, "World law", Fordham International Law Review, 1995, 18, (5), pp. 1617-1622.

Berman, Paul Schiff, "A pluralist approach to international law", Yale Journal of International Law, 2007, 32, (2), pp. 301-329.

Berman, Paul Schiff, "From international law to law and globalization", Columbia Journal of Transnational Law, 2005, 43, (2), pp. 485-556.

Bories, Clémentine, "Histoire des phénomènes administratifs au-delà de la sphère étatique: tâtonnements et hésitations du droit et/ou de la doctrine”, en Bories, Clémentine (ed.), Un droit administratif global? A global administrative law?, Editions Pedone, París, 2012.

Brown, Garrett Wallace, "Moving from cosmopolitan legal theory to legal practice: models of cosmopolitan law", Legal Studies, 2008, 28, (3), pp. 430-451.

Çali, Başak, "On legal cosmopolitanism: divergences in political theory and international law", Leiden Journal of International Law, 2006, 19, (4), pp. 1149-1163. 
Cançado Trindade, Antonio Augusto, International law for humankind: towards a new jus gentium, Martinus Nijhoff Publishers, Leiden, 2010.

Cançado Trindade, Antonio Augusto, Le droit international pour la personne bumaine, Editions Pedone, París, 2011.

Capps, Patrick, "Natural law and the law of nations", en Orakhelashvili, Alexander (ed.), Reseach handbook on the theory and history of international law, Edward Elgar Publishing, Cheltenham, 2011.

Casini, Lorenzo, "Beyond drip-painting? Ten years of GAL and the emergence of a global administration", International Journal of Constitutional Law, 2015, 13, (20), pp. 473-477.

Casini, Lorenzo, "Global administrative law scholarship", en Cassese, Sabino (ed.), Research handbook on global administrative law, Edward Elgar Publishing, Cheltenham, 2016.

Cassese, Sabino, "Administrative law in the global space", en Bories, Clémentine (ed.), Un droit administratif global? A global administrative law?, Editions Pedone, París, 2012.

Cassese, Sabino, "Administrative law without the state - The challenge of global regulation", New York University Journal of International Law and Politics, 2005, 37, (4), pp. 663-694.

Cassese, Sabino, "Global administrative law: the state of the art", International Journal of Constitutional Law, 2015, 13, (2), pp. 465-468.

Cassese, Sabino, "Global standards for national administrative procedure", Law and Contemporary Problems, 2005, 68, (3-4), pp. 109-126.

Cassese, Sabino, "Is there a global administrative law?", en Von Bogdandy, Armin, Wolfrum, Rüdiger, von Bernstorff, Jochen, Dann, Philipp \& Goldmann, Matthias (eds.), The exercise of public authority by international institutions: advancing international institutional law, Springer, Heidelberg, 2010.

Cassese, Sabino, "The globalization of law", New York University Journal of International Law and Politics, 2006, 37, (4), pp. 973-993.

Cassese, Sabino, Carotti, Bruno, Casini, Lorenzo et al. (eds.), Global administrative law: the casebook, Istituto di Ricerche sulla Pubblica Amministrazione-Institute for International Law and Justice, RomaNueva York, 2012.

Cassese, Sabino, Chi governa il mondo?, Il Mulino, Bologna, 2013.

Cassese, Sabino, Oltre lo stato, Editori Laterza, Roma, 2006.

Cassese, Sabino, The global polity: global dimensions of democracy and the rule of law, Global Law Press, Sevilla, 2012. 
Chen, Lung-chu, "Perspectives from the New Haven School", American Society of International Law Proceedings, 1993, (87), pp. 407-411.

Chimni, Bhupinder Singh, "Co-option and resistance: two faces of global administrative law", New York University Journal of International Law and Politics, 2005, 37, (4), pp. 799-827.

Chimni, Bhupinder Singh, "Third World approaches to international law: a manifesto", International Community Law Review, 2006, 8, (1), pp. 3-27.

Chiti, Edoardo, "Where does GAL find its legal grounding?", International Journal of Constitutional Law, 2015, 13, (2), pp. 486-491.

Cover, Robert, “The folktales of justice: tales of jurisdiction", Capital University Law Review, 1985, 14, (2), pp. 179-203.

Cover, Robert, "The Supreme Court, 1982 term - Foreword: nomos and narrative", Harvard Law Review, 1983, 97, (1), pp. 4-68.

Cover, Robert, "The uses of jurisdictional redundancy: interest, ideology, and innovation", William and Mary Law Review, 1981, 22, (4), pp. 639-682.

Dann, Philipp \& Von Engelhardt, Marie, "Legal approaches to global governance and accountability: informal lawmaking, international public authority, and global administrative law compared", en Pauwelyn, Joost, Wessel, Ramses \& Wouters, Jan (eds.), Informal international lawmaking, Oxford University Press, Oxford, 2012.

D'Aspremont, Jean, “Cognitive conflicts and the making of international law: from empirical concord to conceptual discord in legal scholarship", Vanderbilt Journal of Transnational Law, 2013, 46, (4), pp. 1119-1147.

D'Aspremont, Jean, "Droit administratif global et droit international", en Bories, Clémentine (ed.), Un droit administratif global? A global administrative law?, Editions Pedone, París, 2012.

Del Vecchio, Giorgio, "Toward a world law", Boston University Law Review, 1962, 42, (2), pp. 155-159.

Domingo, Rafael, ¿Qué es el derecho global?, Editorial Aranzadi, Cizur Menor, Navarra, 2008.

Domingo, Rafael, "Gaius, Vattel, and the new global law paradigm", European Journal of International Law, 2011, 22, (3), pp. 627-647.

Domingo, Rafael, "The crisis of international law", Vanderbilt Journal of Transnational Law, 2009, 42, (5), pp. 1543-1593.

Domingo, Rafael, "The new global human community", Chicago Journal of International Law, 2012, 12, (2), pp. 563-587. 
Domingo, Rafael, The new global law, Cambridge University Press, Nueva York, 2010.

Dubin, Laurence, "Le droit administratif global, analyse critique de son existence et son articulation avec le droit international public", en Bories, Clémentine (ed.), Un droit administratif global? A global administrative law?, Editions Pedone, París, 2012.

Dyzenhaus, David, "Accountability and the concept of (global) administrative law", Acta Juridica, 2009, (1), pp. 3-31.

Falk, Richard, "Casting the spell: the New Haven School of International Law", Yale Law Journal, 1995, 104, (7), pp. 1991-2008.

Fassbender, Bardo, The United Nations Charter as the constitution of the international community, Martinus Nijhoff Publishers, Leiden, 2009.

Feldman, Noah, "Cosmopolitan law?", Yale Law Journal, 2007, 116, (5), pp. 1022-1070.

Fine, Robert, Cosmopolitanism, Routledge, Abingdon-on-Thames, 2007.

Forteau, Mathias, "Le droit administratif global, signe d'une evolution des techniques du droit international?", en Bories, Clémentine (ed.), Un droit administratif global? A global administrative law?, Editions Pedone, París, 2012.

Fuller, Lon, The morality of law, Yale University Press, New Haven, Ст, 1964. Gathii, James Thuo, "TWAIL: a brief history of its origins, its decentralized network and a tentative bibliography", Trade, Law and Development, 2011, 3, (1), pp. 26-64.

Glazewski, Jan \& Rumble, Olivia, "A rose is a rose but is an 'acacia' an 'acacia'? Global administrative law in action”, Acta Juridica, 2009, (1), pp. 381-402.

Gordon, Geoffrey, "The innate cosmopolitan tradition of international law", Cambridge Journal of International and Comparative Law, 2013, 2, (4), pp. 906-934.

Guilmain, Antoine, "Du droit cosmopolitique au droit global: pour une rupture epistemologique dans l'approche juridique", Revue Quebecoise de Droit International, 2014, 26, (2), pp. 219-236.

Gunton, Dave, Livermore, Mike, Tzanakopoulos, Antonios, et al. "A global administrative law bibliography", Law and Contemporary Problems, 2005, 68, (3-4), pp. 357-377.

Harlow, Carol, "Global administrative law: the quest for principles and values”, European Journal of International Law, 2006, 17, (1), pp. 187-213.

Harlow, Carol, "Voices of difference in a plural community", American Journal of Comparative Law, 2002, 50, (2), pp. 339-367. 
Hart, Herbert Lionel Adolphus, The concept of law, Oxford University Press, Oxford, 1961.

Hathaway, Oona, "The continuing influence of the New Haven School", Yale Journal of International Law, 2007, 32, (2), pp. 553-558.

Hennebel, Ludovic, "Penser le droit administratif global. Scepticisme, idealisme et pragmatisme", en Bories, Clémentine (ed.), Un droit administratif global? A global administrative law?, Editions Pedone, París, 2012.

Jessup, Philip, "Diversity and uniformity in the law of nations", American Journal of International Law, 1964, 58, (2), pp. 341-358.

Jessup, Philip, Transnational law, Yale University Press, New Haven, Ст, 1956.

Kant, Immanuel, 'Toward perpetual peace' and other writings on politics, peace, and history, Yale Unversity Press, New Haven, 2006.

Kant, Immanuel, Hacia la pazperpetua. Un ensayo filosófico, Editorial Biblioteca Nueva, Madrid, 2016.

Kant, Immanuel, Zum ewigen frieden. Ein philosophischer entwurf, Friedrich Nicolovius, Königsberg, 1795.

Kingsbury, Benedict, "Neo-madisonian global constitutionalism: Thomas M. Franck's democratic cosmopolitan prospectus for managing diversity and world order in the twenty-first century", New York University Journal of International Law and Politics, 2003, 35, (2), pp. 291-301.

Kingsbury, Benedict, "The administrative law frontier in global governance", American Society of International Law Proceedings, 2005, (99), pp. 143-153.

Kingsbury, Benedict, "The concept of 'law' in global administrative law", European Journal of International Law, 2009, 20, (1), pp. 23-57.

Kingsbury, Benedict, "Weighing global regulatory rules and decisions in national courts", Acta Juridica, 2009, (1), pp. 90-119.

Kingsbury, Benedict, Krisch, Nico, Stewart, Richard \& Wiener, Jonathan, "Global governance as administration - National and transnational approaches to global administrative law", Law and Contemporary Problems, 2005, 68, (3-4), pp. 1-13.

Kingsbury, Benedict, Krisch, Nico \& Stewart, Richard, "El surgimiento del derecho administrativo global", Revista de Derecho Público (de la Universidad de los Andes, Colombia), 2010, (24), pp. 4-46.

Kingsbury, Benedict, Krisch, Nico \& Stewart, Richard, 'L'emergence d'un droit administratif global", en Bories, Clémentine (ed.), Un droit administratif global? A global administrative law?, Editions Pedone, París, 2012. 
Kingsbury, Benedict, Krisch, Nico \& Stewart, Richard, "The emergence of global administrative law", Law and Contemporary Problems, 2005, 68, (3-4), pp. 15-61.

Kingsbury, Benedict \& Casini, Lorenzo, "Global administrative law dimensions of international organizations law", International Organizations Law Review, 2009, 6, (2), pp. 319-358.

Kingsbury, Benedict \& Donaldson, Megan, "Global administrative law", en Wolfrum, Rüdiger (ed.), Max Planck encyclopedia of public international law, Oxford University Press, Oxford, 2015.

Klabbers, Jan, An introduction to international organizations law, Cambridge University Press, Cambridge, 2015.

Klabbers, Jan, Peters, Anne \& Ulfstein, Geir, The constitutionalization of international law, Oxford University Press, Oxford, 2011.

Koch, Charles, "Introduction: globalization of administrative and regulatory practice", Administrative Law Review, 2002, 54, (1), pp. 409-414.

Koh, Harold Hogjou, "Is there a 'new' New Haven School of International Law?", Yale Journal of International Law, 2007, 32, (2), pp. 559-573.

Koh, Harold Hogjou, "Why transnational law matters", Penn State International Law Review, 2006, 24, (4), pp. 745-753.

Koh, Harold Hongjou, "Bringing international law home", Houston Law Review, 1998, 35, (3), pp. 623-681.

Koh, Harold Hongjou, "How is international human rights law enforced?", Indiana Law Journal, 1999, 74, (4), pp. 1397-1417.

Koh, Harold Hongjou, "The globalization of freedom", Yale Journal of International Law, 2001, 26, (2), pp. 305-312.

Koh, Harold Hongjou, "Transnational legal process", Nebraska Law Review, 1996, 75, (1), pp. 181-207.

Koh, Harold Hongjou, "Why do nations obey international law?", Yale Law Journal, 1997, 106, (8), pp. 2599-2659.

Koskenniemi, Martti, "Legal cosmopolitanism: Tom Franck's messianic world", New York University Journal of International Law and Politics, 2003, 35, (2), pp. 471-486.

Koskenniemi, Martti, The gentle civilizer of nations. The rise and fall of international law 1870-1960, Cambridge University Press, Cambridge, 2005.

Krisch, Nico, "Global administrative law and the constitutional ambition", en Dobner, Petra \& Loughlin, Martin (eds), The twilight of constitutionalism?, Oxford University Press, Oxford, 2010.

Krisch, Nico, "Global governance as public authority: an introduction", International Journal of Constitutional Law, 2012, 10, (4), pp. 976-987. 
Krisch, Nico, "The pluralism of global administrative law", European Journal of International Law, 2006, 17, (1), pp. 247-278.

Krisch, Nico \& Kingsbury, Benedict, "Global governance and global administrative law in the international legal order", European Journal of International Law, 2006, 17, (1), pp. 1-13.

Kunz, Josef, "The 'Vienna School' and international law", New York University Law Quarterly Review, 1934, 11, (3), pp. 370-421.

Kuo, Ming-Sung, "Between fragmentation and unity: the uneasy relationship between global administrative law and global constitutionalism", San Diego International Law Journal, 2009, 10, (2), pp. 439-467.

Kuo, Ming-Sung, "Taming governance with legality - Critical reflections upon global administrative law as small-C global constitutionalism", New York University Journal of International Law and Politics, 2011, 44, (1), pp. 55-102.

Kuo, Ming-Sung, "The concept of 'law' in global administrative law: a reply to Benedict Kingsbury", European Journal of International Law, 2010, 20, (4), pp. 997-1004.

Lador-Lederer, Josef, 'Some observations on the 'Vienna School' in International Law", Netherlands International Law Review, 1970, 17, (2), pp. $126-150$

Lasswell, Harold \& McDougal, Myres, Jurisprudence for a free society: studies in law, science and policy, Martinus Nijhoff Publishers-New Haven Press, Dordrecht-New Haven, 1992.

Luhmann, Niklas, Soziale systeme: Grundriß einer allgemeinen theorie, Suhrkamp Verlag, Frankfurt, 1984.

Marks, Susan, "Naming global administrative law", New York University Journal of International Law and Politics, 2005, 37, (4), pp. 995-1001.

McLean, Janet, "Divergent legal conceptions of the state: implications for global administrative law", Law and Contemporary Problems, 2005, 68, (3-4), pp. 167-187.

Mockle, Daniel, "Le debat sur les principes et les fondements du droit administratif global", Cahiers de Droit, 2012, 53, (1), pp. 3-48.

Möllers, Christoph, "Ten years of global administrative law", International Journal of Constitutional Law, 2015, 13, (2), pp. 469-472.

Neff, Stephen, Justice among nations: a history of international law, Harvard University Press, Cambridge, MA, 2014.

Oliver, Covey, "Philip C. Jessup's continuing contribution to international law”, Columbia Law Review, 1962, 62, (7), pp. 1132-1137. 
Orakhelashvili, Alexander, "International law, international politics and ideology", en Orakhelashvili, Alexander (ed.), Research handbook on the theory and history of international law, Edward Elgar Publishing, Cheltenham, 2011.

Pauwelyn, Joost, "Informal international lawmaking: framing the concept and research questions", en Pauwelyn, Joost, Wessel, Ramses \& Wouters, Jan (eds.), Informal international lawmaking, Oxford University Press, Oxford, 2012.

Pauwelyn, Joost, Wessel, Ramses \& Wouters, Jan (eds.), Informal international lawmaking, Oxford University Press, Oxford, 2012.

Pauwelyn, Joost, Wessel, Ramses \& Wouters, Jan, "When structures become shackles: stagnation and dynamics in international lawmaking", European Journal of International Law, 2014, 25, (3), pp. 733-763.

Reisman, Michael, "International lawmaking: a process of communication", American Society of International Law Proceedings, 1981, (75), pp. 101-120.

Reisman, Michael, "The view from the New Haven School of International Law", American Society of International Law Proceedings, 1992, (86), pp. 118-125.

Reisman, Michael, "Theory about law: jurisprudence for a free society", Yale Law Journal, 1999, 108, (5), pp. 935-939.

Reisman, Michael, Wiessner, Siegfried \& Willard, Andrew, "New Haven School: a brief introduction", Yale Journal of International Law, 2007, 32, (2), pp. 575-582.

Rudall, Jason, "A cartography of cosmopolitanism: particularising the universal", Cambridge Journal of International and Comparative Law, 2014, 3, (3), pp. 747-769.

Sato, Yoshiaki, "Towards the institutionalization of cosmopolitan lawmaking", Alberta Law Review, 2009, 46, (4), pp. 1141-1160.

Savino, Mario, "What if global administrative law is a normative project?", International Journal of Constitutional Law, 2015, 13, (2), pp. 492-498.

Schachter, Oscar, "Philip Jessup's life and ideas", American Journal of International Law, 1986, 80, (4), pp. 878-895.

Schermers, Henry \& Blokker, Niels, International institutional law, Martinus Nijhoff Publishers, Leiden, 2011.

Schill, Stephan, "Lex mercatoria”, en Wolfrum, Rüdiger (ed.), Max Planck encyclopedia of public international law, Oxford University Press, Oxford, 2015.

Simma, Bruno, "From bilateralism to community interest in international law”, Recueil des Cours, 1994/VI, (250), pp. 217-384. 
Somek, Alexander, “Administration without sovereignty”, en Dobner, Petra \& Loughlin, Martin (eds.), The twilight of constitutionalism?, Oxford University Press, Oxford, 2010.

Somek, Alexander, "The concept of 'law' in global administrative law: a reply to Benedict Kingsbury", European Journal of International Law, 2010, 20, (4), pp. 985-995.

Stewart, Richard, "The global regulatory challenge to U.S. administrative law", New York University Journal of International Law and Politics, 2005, 37, (4), pp. 695-762.

Stewart, Richard, "The normative dimensions and performance of global administrative law", International Journal of Constitutional Law, 2015, 13, (2), pp. 499-506.

Stewart, Richard, "U.S. administrative law: a model for global administrative law?", Law and Contemporary Problems, 2005, 68, (3-4), pp. 63-108.

Suzuki, Eisuke, "The New Haven School of International Law: an invitation to a policy-oriented jurisprudence", Yale Studies in World Public Order, 1974, (1), pp. 1-48.

Tesón, Fernando, "The Kantian theory of international law", Columbia Law Review, 1992, 92, (1), pp. 53-102.

Urueña, René, "Global administrative law and the global south", en Cassese, Sabino (ed.), Research handbook on global administrative law, Edward Elgar Publishing, Cheltenham, 2016.

Verdross, Alfred, Der verfassung der völkerrechtsgemeinschaft, Springer-Verlag, Berlín, 1926.

Von Bogdandy, Armin, Dann, Philipp \& Goldmann, Matthias, "Developing the publicness of public international law: towards a legal framework for global governance activities", German Law Journal, 2008, 9, (11), pp. 1375-1400.

Von Bogdandy, Armin, Dann, Philipp \& Goldmann, Matthias, "Developing the publicness of public international law: towards a legal framework for global governance activities", en Von Bogdandy, Armin, Wolfrum, Rüdiger, Von Bernstorff, Jochen, Dann, Philipp \& Goldmann, Matthias (eds.), The exercise of public authority by international institutions: advancing international institutional law, Springer, Heidelberg, 2010.

Von Bogdandy, Armin, Wolfrum, Rüdiger, Von Bernstorff, Jochen, Dann, Philipp \& Goldmann, Matthias (eds.), The exercise of public authority by international institutions: advancing international institutional law, Springer, Heidelberg, 2010. 
Von Bogdandy, Armin \& Venzke, Ingo (eds.), International judicial lawmaking, Springer, Heidelberg, 2012.

Weiler, Joseph, "GAL at a crossroads: preface to the symposium", International Journal of Constitutional Law, 2015, 13, (2), pp. 463-464.

Wiessner, Siegfried, "The New Haven School of Jurisprudence: a universal toolkit for understanding and shaping the law", Asia Pacific Law Review, 2010, 18, (1), pp. 45-61.

Wiessner, Siegfried \& Willard, Andrew, "Policy-oriented jurisprudence", German Yearbook of International Law, 2001, (44), pp. 96-112.

Zumbansen, Peer, "Transnational law", en Smits, Jan (ed.), Elgar encyclopedia of comparative law, Edward Elgar Publishing, Cheltenham, 2006. 
